\title{
Mechanotransduction in mouse inner ear hair cells requires transmembrane channel-like genes
}

\author{
Yoshiyuki Kawashima, ${ }^{1}$ Gwenaëlle S.G. Géléoc, ${ }^{2,3}$ Kiyoto Kurima, ${ }^{1}$ Valentina Labay, ${ }^{1}$ \\ Andrea Lelli, ${ }^{2}$ Yukako Asai, ${ }^{2}$ Tomoko Makishima, ${ }^{1}$ Doris K. Wu, ${ }^{4}$ \\ Charles C. Della Santina, ${ }^{5}$ Jeffrey R. Holt, ${ }^{2,3}$ and Andrew J. Griffith ${ }^{1}$
}

\begin{abstract}
${ }^{1}$ Molecular Biology and Genetics Section, National Institute on Deafness and Other Communication Disorders (NIDCD), NIH, Rockville, Maryland, USA. 2Departments of Neuroscience and Otolaryngology, University of Virginia School of Medicine, Charlottesville, Virginia, USA.

3Department of Otolaryngology, Children's Hospital Boston, and Harvard Medical School, Boston, Massachusetts, USA.

${ }^{4}$ Section on Sensory Cell Regeneration and Development, NIDCD, NIH, Rockville, Maryland, USA.

${ }^{5}$ Department of Otolaryngology — Head and Neck Surgery, Johns Hopkins University School of Medicine, Baltimore, Maryland, USA.
\end{abstract}

\begin{abstract}
Inner ear hair cells convert the mechanical stimuli of sound, gravity, and head movement into electrical signals. This mechanotransduction process is initiated by opening of cation channels near the tips of hair cell stereocilia. Since the identity of these ion channels is unknown, and mutations in the gene encoding transmembrane channel-like 1 (TMC1) cause hearing loss without vestibular dysfunction in both mice and humans, we investigated the contribution of $T m c 1$ and the closely related $T m c 2$ to mechanotransduction in mice. We found that $T m c 1$ and $T m c 2$ were expressed in mouse vestibular and cochlear hair cells and that GFP-tagged TMC proteins localized near stereocilia tips. Tmc 2 expression was transient in early postnatal mouse cochlear hair cells but persisted in vestibular hair cells. While mice with a targeted deletion of $\operatorname{Tmc1}\left(T m c 1^{\Delta}\right.$ mice) were deaf and those with a deletion of $T m c 2$ ( $T m c 2^{\Delta}$ mice) were phenotypically normal, $T m c 1^{\Delta} T m c 2^{\Delta}$ mice had profound vestibular dysfunction, deafness, and structurally normal hair cells that lacked all mechanotransduction activity. Expression of either exogenous TMC1 or TMC2 rescued mechanotransduction in $T m c 1^{\Delta} T m c 2^{\Delta}$ mutant hair cells. Our results indicate that TMC1 and TMC2 are necessary for hair cell mechanotransduction and may be integral components of the mechanotransduction complex. Our data also suggest that persistent TMC2 expression in vestibular hair cells may preserve vestibular function in humans with hearing loss caused by TMC1 mutations.
\end{abstract}

\section{Introduction}

Dominant and recessive mutations of human transmembrane channel-like 1 (TMC1) cause DFNA36 hearing loss and DFNB7/ B11 deafness, respectively (1). Similarly, semidominant and recessive alleles of Tmc1 cause hearing loss in Beethoven (Bth) and deafness $(d n)$ mutant mice $(1,2)$. TMC1 is a member of the novel TMC gene family that includes 7 other paralogs in mammals $(3,4)$. The function of TMC genes remains obscure, and the in silico translation products have no significant sequence similarity to proteins or domains of known function; however, all are predicted to encode integral membrane proteins with at least 6 membranespanning domains $(3,4)$. This 6-pass transmembrane topology has been experimentally confirmed for mouse TMC1 expressed in heterologous systems (5) and suggests it may function as a receptor, pump, transporter, or channel $(3,4)$.

In mouse, Tmc1 and the closely related $T m c 2$ are expressed at low levels in a restricted tissue distribution (1-3). Tmc1 mRNA is expressed in auditory and vestibular hair cells $(1,2)$, but there is no evidence of vestibular dysfunction associated with TMC1 or Tmc1

Authorship note: Yoshiyuki Kawashima, Gwenaëlle S.G. Géléoc, and Kiyoto Kurima are co-first authors. Jeffrey R. Holt and Andrew J. Griffith are co-senior authors.

Conflict of interest: Charles C. Della Santina owns equity in Labyrinth Devices LLC. Kiyoto Kurima and Andrew J. Griffith hold the following U.S. patents: 7,166,433 (Transductin-2 and Applications to Hereditary Deafness), 7,192,705 (Transductin-1 and Applications to Hereditary Deafness), and 7,659,115 (Nucleic Acid Encoding Human Transductin-1 Polypeptide)

Citation for this article: JClin Invest. 2011;121(12):4796-4809. doi:10.1172/JCI60405. mutations $(1,2,6-8)$. Tmc2 mRNA is also detectable in mouse inner ear (1), but neither TMC2 nor Tmc2 has been implicated in inner ear dysfunction. These observations could reflect incomplete penetrance of mutant phenotypes, functional redundancy of $T m c 1$ and $T m c 2$, differential requirements for $T m c 1$ versus $T m c 2$ in auditory and vestibular hair cells, or a combination of these mechanisms.

Marcotti et al. (9) investigated the function of Tmc1 in auditory hair cells excised from $T m c 1^{d n}$ and $T m c 1^{\text {Bth }}$ mice and suggested that $T m c 1$ is necessary for hair cell maturation but is not required for mechanotransduction, the process by which hair cells convert mechanical stimuli into electrical signals. Mechanotransduction is initiated near the tips of stereocilia (10-14) on the apical surface of hair cells and is mediated by an unidentified ion channel gated by mechanical force (15). Hair cell mechanotransduction is characterized by large single-channel conductances of approximately 100 pS (16-18), rapid activation with sub-millisecond kinetics $(19,20)$, and nonselective permeability to cations (19) and other larger molecules (21) including the fluorescent dye FM1-43 (22, 23) and aminoglycoside antibiotics (24). There are several known pharmacologic agents that inhibit hair cell mechanotransduction, but a selective, high-affinity antagonist has not been identified (25). The difficulty in identifying the hair cell mechanotransduction channel at the molecular level could arise from functional pleiotropy, functional redundancy, or both (26). Here we provide evidence supporting the novel hypothesis that TMC1 and TMC2 are functionally redundant stereocilia components necessary for hair cell mechanotransduction. 
A

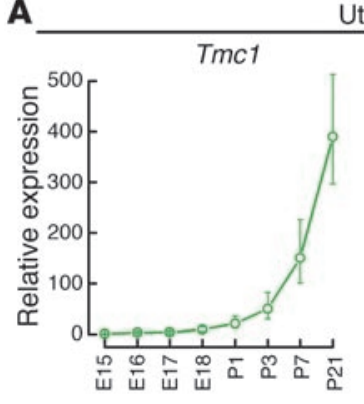

C

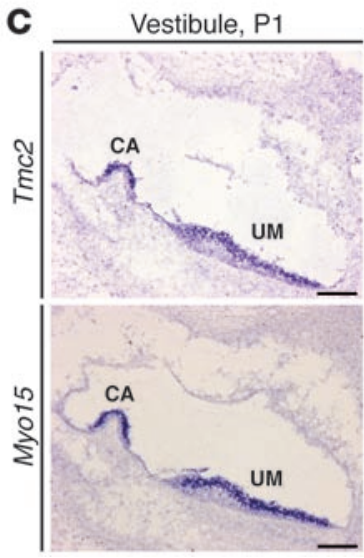

Utricle

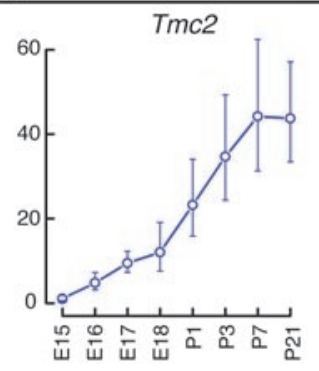

B

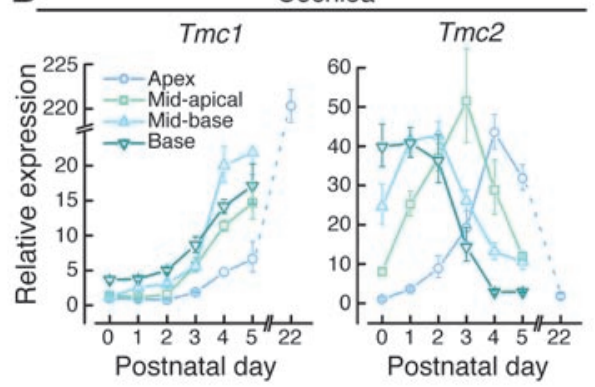

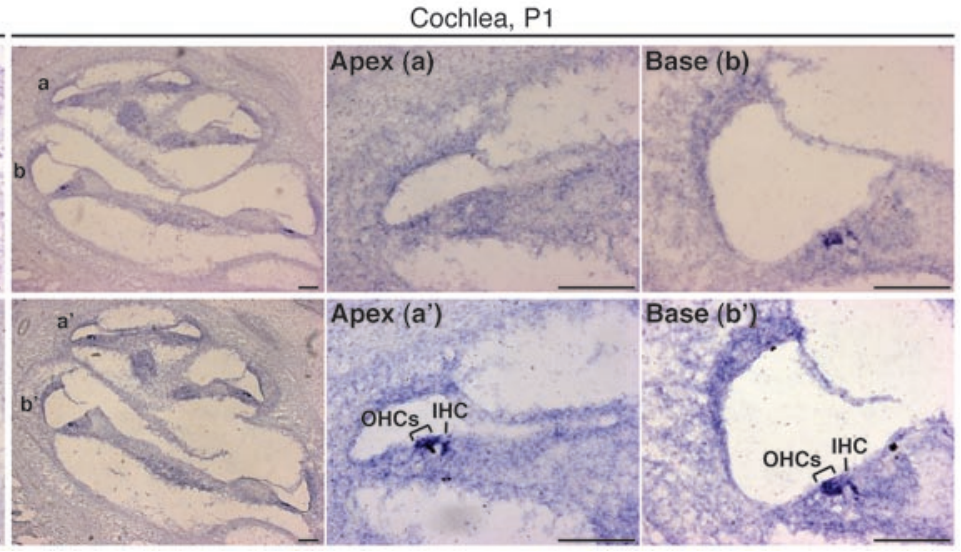

\section{Figure 1}

Hair cell expression of Tmc1 and Tmc2. (A) qPCR analysis of Tmc1 and Tmc2 in RNA extracted from mouse utricles at developmental ages between E15 and P21. We used primers that amplify a fragment of $T m c 1$ common to both $T m c 1$ ex1 and Tmc1ex2. Each sample was analyzed $6-9$ times. Expression levels were normalized, using the $\Delta \Delta \mathrm{Ct}$ method, first to Actb (encoding $\beta$-actin) expression and then to the Tmc expression level at E15. Error bars indicate \pm SD. (B) qPCR analysis of mouse cochlear RNA showed a steady rise in Tmc1 expression through P5 and in the P22 apical sample. Other portions of P22 cochleae were not harvested due to rapid degradation of hair cell integrity and mRNA quality. Tmc2 RNA was transiently expressed in mouse cochleae during the postnatal period. Error bars indicate SD. (C) In situ hybridization analysis of Tmc2 in P1 mouse vestibule and cochlea. Tmc2 expression was detected in wild-type crista ampullaris (CA), utricular macula (UM), hair cells of the basal cochlear turn, and, to a lesser extent, in the middle turn at P1. Tmc2 expression was not detected in hair cells of the apical turn. Adjacent sections probed for Myo15 transcripts are shown as a control for hair cell hybridization (52). Scale bar: $100 \mu \mathrm{m}$. See also Supplemental Figure 1.

\section{Results}

Tmc1 and Tmc2 expression. Mouse utricular maculae and cochleae express two splice isoforms of Tmc1, Tmc1 $1^{e x 1}$ and $T m c 1^{e x 2}$, which exclude or include exon 2 and encode different translation initiation codons in exons 1 and 2, respectively (Supplemental Figure 1A; supplemental material available online with this article; doi:10.1172/JCI60405DS1). Levels of $T m c 1^{\text {ex1 }}$ and $T m c 1^{\text {ex2 }}$ RNA were similar to each other in either utricular maculae or cochleae excised at P0, P5, or P14 (data not shown).

We performed quantitative RT-PCR (qPCR) analysis of Tmc1 and Tmc2 RNA from mouse utricular maculae and cochleae harvested at different embryonic and postnatal time points (Figure 1, A and B). The primer pair used for Tmc1 qPCR analysis amplified both $T m c 1^{\text {ex } 1}$ and $T m c 1^{e x 2}$. In utricular maculae, a rise in $T m c 2$ expression (Figure 1A) coincided with the onset of mechanotransduction between E16 and E17 (27). The rise in Tmc1 expression (Figure 1A) lagged mechanotransduction acquisition by several days. The levels of Tmc1 and Tmc2 mRNA remained elevated at P21, the last time point tested.

In cochleae, within 5 days after birth, Tmc1 and Tmc2 mRNA levels increased 20-fold and 40-fold, respectively (Figure 1B). The rise in Tmc2 expression (Figure 1B) coincided with the spatiotemporal onset of mechanotransduction in outer hair cells (OHCs) in the basal turn at P0 and the apical turn at P2 (28). The rise in Tmc1 expression (Figure 1B) lagged mechanotransduction acquisition by $2-3$ days in all regions of the cochlea (28). Tmc2 mRNA was transiently expressed during the first postnatal week, while the Tmc1 transcript level increased more than 200-fold by P22. The transient Tmc2 expression profile was confirmed in a second independent sample of cochleae harvested from mice that ranged in age from E17 to P8 (Supplemental Figure 1B).

We also performed in situ hybridization analysis of Tmc2 to identify which specific cell type(s) expressed Tmc2 mRNA (Figure 1C). Tmc2 expression was confirmed in hair cells of the mouse utricle as well as other vestibular sensory organs at P1 (Figure 1C) and P5 (data not shown). Tmc2 expression was detected in inner hair cells (IHCs) and OHCs in basal and middle turns of cochleae excised at P1, but not in the apical turns (Figure 1C), consistent with our qPCR analysis. By P5, Tmc2 expression in the cochlea was below the level of detection using in situ hybridization. These data demonstrate a switch from $T m c 2$ to $T m c 1$ mRNA expression in postnatal cochlear hair cells. 
A

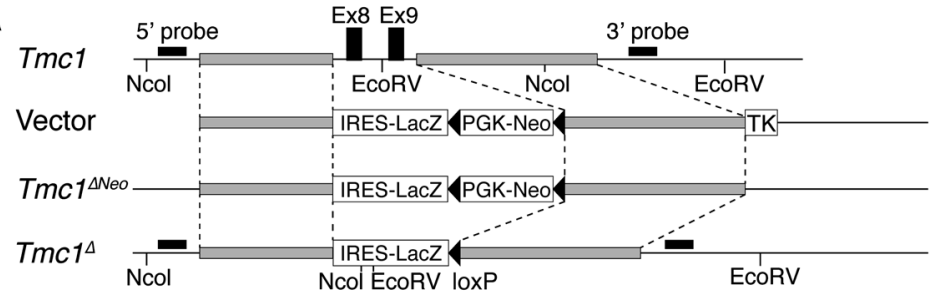

B

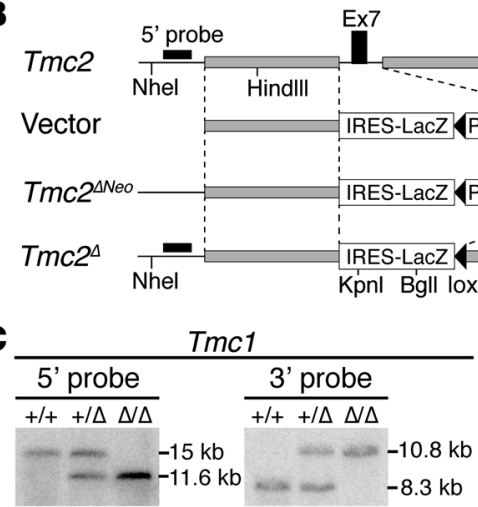

3' probe

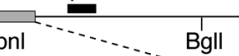

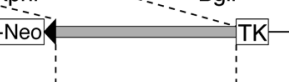

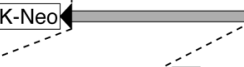

D

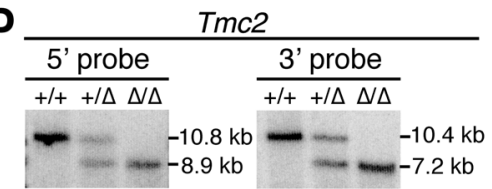

\section{Figure 2}

$T m c 1^{\Delta}$ and $T m c 2^{\Delta}$ mice. (A) Generation of $T m c 1^{\Delta}$ mice. Schematic diagram of the Tmc1 genomic locus, targeting vector, and targeted locus before and after Cremediated excision of PGK-Neo. 5' and 3' hybridization probes and restriction digestion sites for Southern blot analysis are indicated. TK encodes thymidine kinase. (B) Generation of $T m c 2^{\Delta}$ mice. (C) Southern blot analyses of Tmc1 targeting. Genomic DNA was digested with $\mathrm{Ncol}$ for $5^{\prime}$ probe hybridization and EcoRV for 3' probe hybridization. (D) Southern blot analyses of Tmc2 targeting. Genomic DNA was digested with Nhel and Kpnl for $5^{\prime}$ probe hybridization and Bgll and Hindlll for $3^{\prime}$ probe hybridization. The sizes of the $T m c 1^{\Delta}$ and $T m c 2^{\Delta}$ hybridizing bands confirmed proper integration of the targeting constructs. See also Supplemental Figure 2.
$T m c 1^{\Delta}$ and $T m c 2^{\Delta}$ mice. To generate the $T m c 1^{\Delta}$ and $T m c 2^{\Delta}$ lines, we used homologous recombination in ES cells to delete exon(s) encoding the predicted first transmembrane domains of $T m c 1$ and $T m c 2$, respectively (Figure 2). The exons were replaced with a gene trap construct fused to a lac $Z$ reporter gene to visualize mRNA expression from the endogenous Tmc1 or Tmc2 promoter (Figure 2, A and B). Both lines were backcrossed to C57BL/6J for 10 or more generations. X-gal staining of $T m c 1^{\Delta /+} \mathrm{P} 28$ vestibular end organs detected $\beta$-galactosidase activity in both type I and type II hair cells of the saccular macula, utricular macula, and cristae ampullaris (Supplemental Figure 2, A and B). In Tmc $2^{\Delta /+} \mathrm{P} 28$ inner ears, $\beta$-galactosidase activity was detected in hair cells of the cristae ampullaris (Supplemental Figure 2A) and was barely detectable in hair cells of the saccular and utricular maculae. To characterize transcripts encoded by the targeted Tmc2 locus, we performed RT-PCR analysis of Tmc $2^{\Delta}$ RNA. We identified a $T m c 2^{\Delta}$ transcript in which a portion of the gene trap cassette was spliced into the flanking exons (6 and 8 ) of Tmc2 (Supplemental Figure 2, C and D). This transcript lacked the lac $Z$ reporter sequence, which may account for the low levels of $\beta$-galactosidase activity in $T m c 2^{\Delta}$ hair cells.

$T m c 1^{\Delta}$ and $T m c 2^{\Delta}$ bearing and balance phenotypes. $T m c 1^{\Delta /+}$ mice developed normally, but $T m c 1^{\Delta / \Delta}$ mice were deaf (Supplemental Figure 3) and exhibited normal motor behavior. Both $T m c 2^{\Delta /{ }^{+}}$and $T m c 2^{\Delta / \Delta}$ mice developed normally without hearing loss (Supplemental Figure 3) and showed normal motor behavior. To detect potential genetic interactions of $T m c 1$ with $T m c 2$, we crossed the $T m c 1^{\Delta}$ and $T m c 2^{\Delta}$ lines to generate mice with each of 8 different $T m c 1 ; T m c 2$ genotypes. All of the mutant mice were viable; however, $T m c 1^{\Delta / \Delta} T m c 2^{\Delta / \Delta}$ mice exhibited overtly abnormal vestibular behaviors: head-bobbing and -arching, ataxic gait, and circling (Supplemental Video 1). Moreover, early postnatal $T m c 1^{\Delta / \Delta} T m c 2^{\Delta / \Delta}$ pups lacked the ability to right from a supine position (Supplemental Video 1).

We employed vestibulo-ocular reflex (VOR) testing during high-acceleration, whole-body rotations in darkness to evaluate hair cell mechanosensitivity in the crista ampullaris of the lateral semicircular canal. VOR testing of $T m c 1^{\Delta / \Delta} T m c 2^{\Delta / \Delta}$ mice at 2-3 months of age showed negligible acceleration gain values that were indistinguishable from those of dead mouse controls (Figure 3, A and B). In contrast, $T m c 1^{\Delta / \Delta} T m c 2^{\Delta /+}$ mice had normal acceleration gain values. Tmc1 appears to modify the $T m c 2^{\Delta / \Delta}$ VOR phenotype, since $T m c 1^{\Delta /+} T m c 2^{\Delta / \Delta}$ mice exhibited intermediate values that were significantly higher $(P=0.0059$, Student's $t$ test) than those of $T m c 1^{\Delta / \Delta} T m c 2^{\Delta / \Delta}$ mice and lower $(P=0.0075)$ than those of wild-type mice. The mean acceleration gain of $T m c 1^{+/+} T m c 2^{\Delta / \Delta}$ mice was also higher $(P=0.0030)$ than that of $T m c 1^{\Delta /+} T m c 2^{\Delta / \Delta}$ mice, but not significantly lower $(P=0.1416)$ than that of wild-type mice. This genetic interaction of Tmc1 with Tmc2 could reflect redundancy of their function(s) in the vestibular system.

Auditory brainstem response (ABR) thresholds measured in young adult mice showed that only $T m c 1^{\Delta / \Delta}$ mice had hearing loss, with no effect of the Tmc2 genotype at any frequency tested (Figure 3C). The profound deafness in mice lacking functional Tmc1 was consistent with data from $T m c 1^{B t h / B t h}$ and $T m c 1^{d n / d n}$ mice $(8,29)$. This indicates that $T m c 1$, but not $T m c 2$, is necessary for normal auditory function in adult mice.

$T m c 1^{\Delta}$ and $\mathrm{Tmc}^{\Delta}$ hair cell structure. We analyzed vestibular and cochlear hair bundle morphology using scanning electron microscopy (SEM). Both vestibular and cochlear $T m c 1^{\Delta / \Delta} T m c 2^{\Delta / \Delta}$ hair cells appeared to have intact hair bundles, tip links, and tenting of tips of shorter stereocilia that were indistinguishable from those of wild-type controls at P3 (Figure 4), when wild-type hair cells have acquired functional mechanotransduction $(27,28)$. Furthermore, vestibular hair bundles appeared normal through 4 months of age (Supplemental Figure 4A). In cochleae, degeneration of $T m c 1^{\Delta / \Delta} T m c 2^{\Delta / \Delta} \mathrm{OHC}$ bundles was first apparent at P5 and P7 in the basal and apical cochlear turns, respectively, while IHC bundles appeared normal until approximately P9 (Supplemental Figure 4B). In contrast, Tmc1 $1^{\Delta / \Delta} \mathrm{Tmc}^{+/+} \mathrm{IHC}$ and OHC bundles developed normally until P14 and then degenerated (Supplemental Figure 4C), consistent with reported observations of 

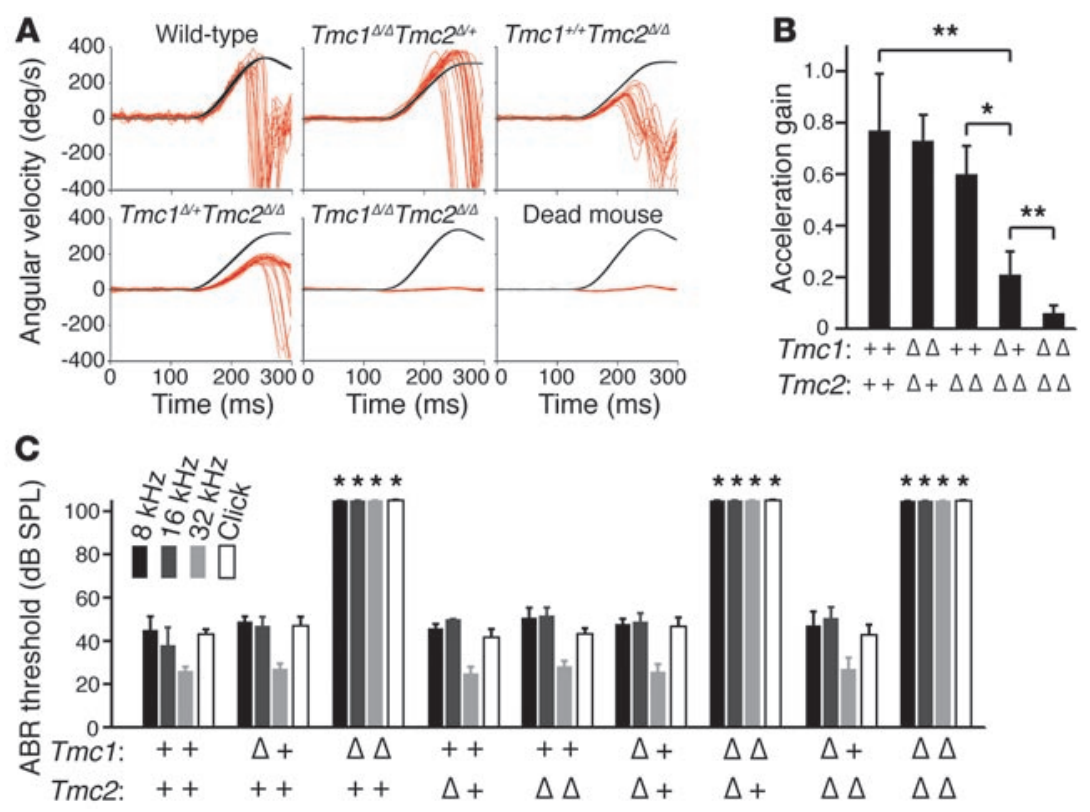

Figure 3

$T m c 1^{\Delta}$ and $T m c 2^{\Delta}$ hearing and balance phenotype. (A) Representative VOR traces. Black traces show head angular velocity (degrees per second [deg/s]) during passive, wholebody rotations delivered in darkness about an Earth-vertical axis with the animal's horizontal semicircular canals in an Earth-horizontal plane. The red traces show angular velocity of the eye during more than 16 VOR-mediated counter-rotations. Head angular velocity is inverted to facilitate visual comparison. Reflexive eye movements during the first $100 \mathrm{~ms}$ of the head rotation stimulus are a sensitive measure of semicircular canal (crista ampullaris) function. $T m c 1^{+++} T m c 2^{\Delta / \Delta}$ and $T m c 1^{\Delta /+} T m c 2^{\Delta / \Delta}$ mice had reduced responses compared with those of a wild-type mouse, whereas the $T m c 1^{\Delta / \Delta} T m c 2^{\Delta / \Delta}$ mouse and a dead mouse control had no response. (B) Mean $( \pm \mathrm{SD})$ acceleration gains in $\operatorname{VOR}\left({ }^{\star} P<0.05,{ }^{\star \star} P<0.01\right.$, Student's $t$ test). (C) Mean ( $\pm \mathrm{SD}$ ) ABR thresholds for tone-burst stimuli of $8 \mathrm{kHz}, 16 \mathrm{kHz}$, or $32 \mathrm{kHz}$, or click stimuli in better-hearing ears of 12-week-old mice. Mice lacking functional Tmc1 had profound hearing loss, with no effect of Tmc2 genotype. ${ }^{*} P<0.001$, 1-way ANOVA, compared with wild-type values. See also Supplemental Figure 3.

$T m c 1^{d n / d n}$ hair cells (8). Tmc $1^{+/+} T m c 2^{\Delta / \Delta}$ cochlear hair bundles appeared normal (Supplemental Figure 4C), as expected for a genotype associated with normal ABR thresholds (Figure 3 and Supplemental Figure 3).

$T m c 1^{\Delta}$ and $T m c 2^{\Delta}$ bair cell function. To examine mechanosensory function in vestibular hair cells of Tmc1;Tmc2 mutant mice, we recorded mechanotransduction currents from P1-P8 hair cells excised from the utricle $(17,30)$, an organ of balance sensitive to linear acceleration. To avoid using hair bundles damaged during dissection, we selected only hair cells with visibly intact stereocilia bundles for analysis. Utricle hair cells that lacked Tmc1 had normal transduction currents (Figure 5, A and B), consistent with the lack of behavioral and VOR phenotypes in those mice. Hair cells that lacked Tmc2, however, had significantly attenuated transduction currents, reduced more than $80 \%$ relative to wild-type controls. In hair cells that lacked both Tmc1 and Tmc2, mechanotransduction currents were completely absent. Hair cells with intermediate genotypes, $T m c 1^{\Delta /+} T m c 2^{\Delta / \Delta}$ and $T m c 1^{\Delta / \Delta} T m c 2^{\Delta /+}$, had reduced current amplitudes relative to wild-type controls $(P<0.001,1$-way ANOVA) (Figure $5 \mathrm{~B}$ ). One or two wild-type alleles of $T m c 2$ yielded approximately $70 \%$ or $100 \%$ of wild-type mechanotransduction current amplitudes, respectively. However, one or two wild-type alleles of Tmc1 yielded no more than $15 \%$ of wild-type mechanotransduction current amplitudes. Data collected from 111 type II utricle hair cells are summarized in Figure 5B. In hair cells with intermediate genotypes, we analyzed the $10 \%-90 \%$ operating range of stimulus-response relationships, but found no significant difference relative to wild-type controls, indicating that the sensitivity of mechanotransduction was unaltered by deletion of Tmc1 or Tmc2. Marcotti et al. (9) reported that auditory hair cells of mice that carried either the $d n$ or Bth mutation in Tmc1 had reduced voltage-dependent currents at adolescent and adult stages (>P14) but normal current amplitudes at earlier postnatal stages $(<\mathrm{P} 7)$. We examined voltage-dependent currents from type II vestibular hair cells of Tmc1;Tmc2 mutant mice at postnatal stages (P5-P7). Consistent with the results of Marcotti et al. (9), we found no difference in current amplitudes, kinetics, or voltage dependence for any of the Tmc1; Tmc2 genotypes examined (Supplemental Figure 5). Thus, the only electrophysiological deficit we detected in Tmc1; Tmc2-deficient utricle hair cells was the absent or reduced mechanotransduction current.

In cochleae, we recorded whole-cell mechanotransduction currents from apical-turn OHCs at P5-P7 as previously described $(28,31)$. OHCs from mice with at least one functional allele of $T m c 1$ had mechanotransduction currents that were indistinguishable from those of wild-type controls, regardless of the Tmc2 genotype (Figure 6, A and B). In contrast to a previous report on $T m c 1^{B t h / B t h} \mathrm{Tmc}^{+/+}$and $T m c 1^{d n / d n} T_{m c 2^{+/+}}$hair cells (9), apical-turn OHCs that lacked both alleles of Tmc1 had transduction currents that were significantly reduced relative to those of wild-type hair cells $(P<0.001,1$-way ANOVA). Double homozygous mutant hair cells with neither Tmc1 nor Tmc2 lacked mechanotransduction currents entirely (Figure 6, A and B). The Tmc $1^{\Delta / \Delta} T m c 2^{\Delta / \Delta}$ OHCs had normal voltage-dependent currents at P5-P7, consistent with the Marcotti et al. (9) data for the equivalent postnatal day. Therefore, the absence of mechanotransduction currents in $T m c 1^{\Delta / \Delta} T m c 2^{\Delta / \Delta}$ apical-turn $\mathrm{OHCs}$ was a specific physiologic deficit.

We also recorded mechanotransduction currents from OHCs excised from the basal end of P2-P4 cochleae. Interestingly, $T m c 1^{\Delta / \Delta} \mathrm{Tmc}^{+/+}$basal-turn OHCs had amplitudes similar to those of wild-type OHCs (Figure 6, C and D). This result contrasted with data recorded from apical cochleae at P5-P7 that revealed a significant reduction in current amplitude in $T m c 1^{\Delta / \Delta} T m c 2^{+/+}$cells. Together with the relatively high expression of $T m c 2$ and relatively low expression of Tmc1 in the wild-type cochlear base at P2, the data suggest that $T m c 2$ is necessary for mechanotransduction and can fully compensate for deletion of Tmc1 in basal-turn hair cells at this early postnatal stage. There was a significant reduction in transduction current amplitudes in $T m c 1^{\Delta / \Delta} T m c 2^{\Delta /+}$ OHCs from P2-P4 basal cochleae, which may reflect haploinsufficiency of Tmc2. Consistent with our data from cochlear apex, we found that basal OHCs that lacked both Tmc1 and Tmc2 had no mechanotransduction currents (Figure 6, C and D). 

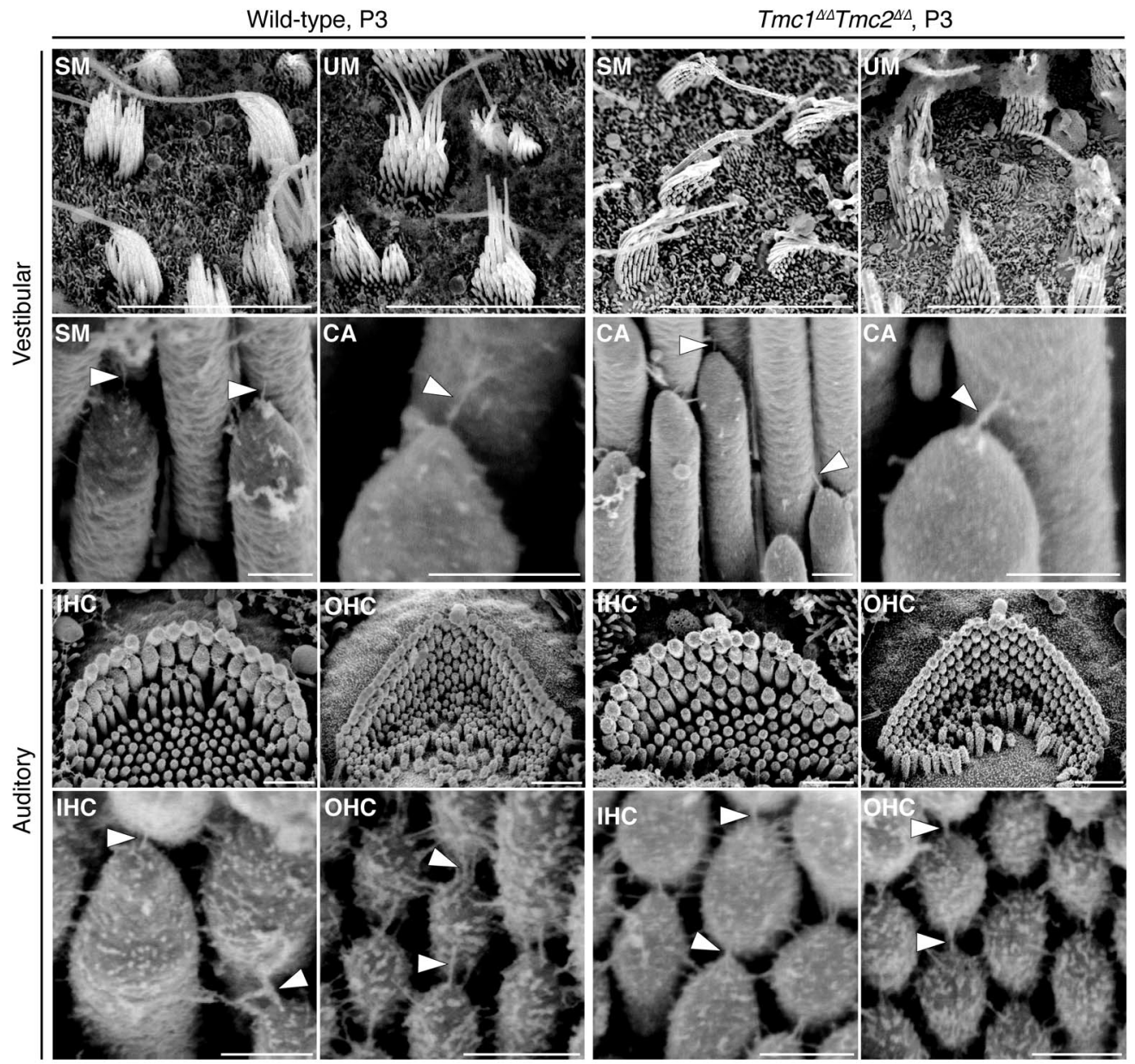

\section{Figure 4}

Hair bundle phenotype of $T m c 1^{\Delta \Delta \Delta}$ and $T m c 2^{\Delta / \Delta}$ mice. Vestibular and auditory hair bundle and tip-link phenotype of P3 wild-type and $T m c 1^{\Delta / \Delta} T m c 2^{\Delta / \Delta}$

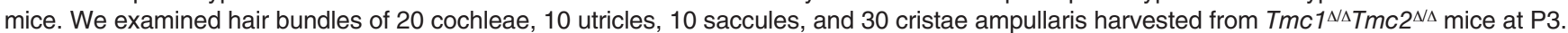
$T m{ }^{1}{ }^{\Delta \Delta} T m 2^{\Delta / \Delta}$ mice had hair bundles and tip links that were indistinguishable from those of wild-type mice. Tenting of tips of shorter stereocilia is a normal indication of tension exerted by tip links. SM, saccular macula; UM, utricular macula; CA, crista ampullaris. Arrowheads indicate tip links. Scale bars: $10 \mu \mathrm{m}$ (top row); $250 \mathrm{~nm}$ (second and fourth rows); $1 \mu \mathrm{m}$ (third row). See also Supplemental Figure 4.

Rescue of $T m c 1^{\Delta / \Delta} T m c 2^{\Delta / \Delta}$ hair cell mechanotransduction with Tmc1 or Tmc2. To investigate whether Tmc1 or Tmc2 is sufficient to restore mechanosensitivity in $T m c 1^{\Delta / \Delta} T m c 2^{\Delta / \Delta}$ hair cells, we generated adenoviral expression vectors encoding $T m c 1^{e x 1}, T m c 1^{e x 2}$, or Tmc2. A fragment of the human MYO7A promoter was initially used to produce relatively low levels of either Tmc1 or Tmc2 expression. The coding sequence for red fluorescent protein (RFP) with a separate promoter was included as a transfection marker. We prepared organotypic cultures from utricles harvested from $T m c 1^{\Delta / \Delta} T m c 2^{\Delta / \Delta}$ mice at P0. All tested hair cells $(n=11)$ that lacked RFP fluorescence also lacked mechanotransduction current (data not shown). $T m c 1^{\Delta / \Delta} T m c 2^{\Delta / \Delta}$ hair cells that were RFP positive, indicating transfection with Ad-Tmc1ex1, AdTmc1 ${ }^{\text {ex2 }}$, or Ad-Tmc2, had mechanotransduction currents (Figure 7, A and B). This suggests that exogenous expression of either Tmc1 or Tmc2 is sufficient to rescue mechanotransduction in $T m c 1^{\Delta / \Delta} T m c 2^{\Delta / \Delta}$ utricle hair cells. We also generated a negative control adenoviral vector, Ad-Tmc1 $1^{\text {exz-dn }}$, encoding the deafness mouse allele of Tmc1 ${ }^{e x 2}$. Hair cells transfected with Ad-Tmc1 $1^{\text {ex2-dn }}$ did not recover mechanotransduction, indicating that rescue of mechanotransduction by TMC1 or TMC2 is a specific effect of expression of functional TMC protein.

We also tested the ability of exogenous TMC1 or TMC2 to rescue mechanotransduction in cochlear cultures harvested from $T m c 1^{\Delta / \Delta} T m c 2^{\Delta / \Delta}$ mice at P0. RFP-positive hair cells that expressed Ad-Tmc2 had mechanotransduction (Figure 7, C and D), while neither Ad-Tmc1 ${ }^{e x 1}$ nor Ad-Tmc1 ${ }^{e x 2}$ rescued mechanotransduction (data not shown). We also generated a $T m c 1^{\text {ext }}$ expression vector driven by a CMV promoter to produce relatively higher levels of Tmc1 expression. RFP-positive hair cells that expressed CMV promoter-driven Ad-Tmc1 ${ }^{x x}$ had unambiguous mechanotransduction currents (Figure 7, C and D), indicating that exogenous expression of either Tmc1 or Tmc2 was sufficient to restore mechanotransduction in $T m c 1^{\Delta / \Delta} T m c 2^{\Delta / \Delta}$ auditory hair cells. The rescue of hair cell transduction in organotypic cultures raises the possibility that $T m c$ gene replacement in neonatal mice may preserve or restore hearing and balance function in Tmc-deficient animals. The inability to rescue mechanotransduction with $M Y O 7 A$ pro- 
A

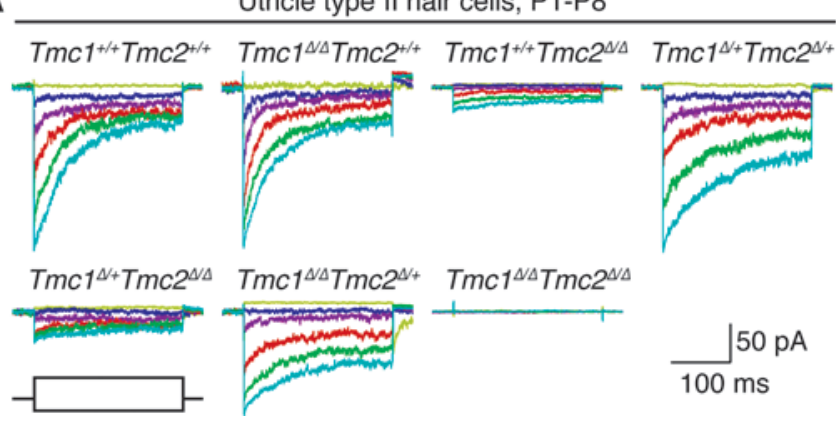

B

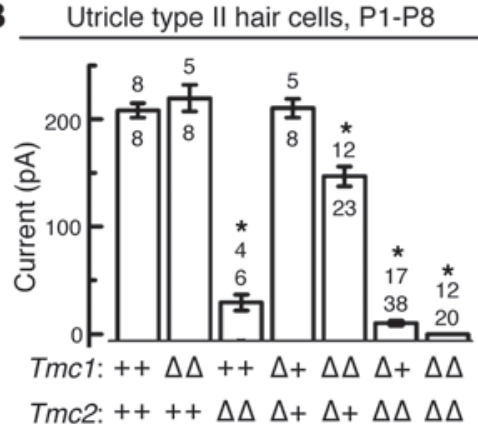

\section{Figure 5}

$T m c 1^{\Delta}$ and $T_{m c 2^{\Delta}}$ vestibular hair cell mechanotransduction currents. (A) Representative mechanotransduction current families for utricle type II hair cells harvested between P1 and P8. Hair bundles were deflected in 250-ms steps that ranged from $-0.5 \mu \mathrm{m}$ to $1 \mu \mathrm{m}$ in $0.1-\mu \mathrm{m}$ increments. The envelope of the stimulus protocol is shown at the bottom left. Currents were recorded at $-64 \mathrm{mV}$, a physiologically relevant holding potential. Some traces have been removed for clarity. Scale bars apply to all current families. (B) Summary bar graph from 111 type II utricle hair cells. The graph shows mean maximal mechanotransduction current amplitudes ( \pm SEM). For each bar, the upper number indicates the number of mice examined, and the lower number indicates the total number hair cells examined for each genotype. ${ }^{*} P<0.001,1$-way ANOVA), relative to wild-type values.

moter-driven Ad-Tmc1 may reflect differences in the TMC1 and TMC2 expression levels required to rescue transduction at the developmental age tested.

Hair cell uptake of FM1-43. We used the styryl membrane dye FM1-43 to visualize mechanotransduction channel activity in thousands of hair cells simultaneously. Intracellular fluorescence following a brief, 10-second exposure to FM1-43 indicates the presence of functional mechanotransduction channels open at rest $(22,23,27)$. Utricles excised from $T m c 1^{\Delta /+} T m c 2^{\Delta /+}$ or $T m c 1^{\Delta / \Delta} T m c 2^{\Delta /+}$ mice at P3 had normal uptake of FM1-43 (Figure 8A). In contrast, utricles from $T m c 1^{\Delta / \Delta} T m c 2^{\Delta / \Delta}$ mice had no detectable FM1-43 uptake. $T m c 1^{\Delta /+} T m c 2^{\Delta / \Delta}$ utricles, with one wild-type allele of Tmc1, showed strong FM1-43 uptake in the peripheral region of the utricle, a pattern reminiscent of the lacZ reporter result for $T m c 1$ expression (Supplemental Figure 2, A and B). Wild-type mouse cochleae excised at P3 had robust FM1-43 uptake in cochlear OHCs and, to a lesser extent, IHCs throughout the cochlea (Figure 8B), as previously reported $(22,28)$. FM1-43 uptake was not detected in any $T m c 1^{\Delta / \Delta} T m c 2^{\Delta / \Delta}$ cochlear hair cells. Since stereocilia tip-link integrity is required for mechanotransduction, we used SEM to evaluate tip links in $T m c 1^{\Delta / \Delta} T m c 2^{\Delta / \Delta}$ cochleae immediately after an FM1-43 uptake experiment. Tip links appeared intact in $T m c 1^{\Delta / \Delta} T m c 2^{\Delta / \Delta} \mathrm{OHC}$ bundles following the dye application protocol (Figure $8 \mathrm{~B}$, insets), indicating the $T m c 1^{\Delta / \Delta} T m c 2^{\Delta / \Delta}$ hair bundles lack some other element(s) essential for FM1-43 uptake and mechanotransduction.

To investigate whether Tmc1 or Tmc2 was sufficient to rescue FM1-43 uptake in $T m c 1^{\Delta / \Delta} T m c 2^{\Delta / \Delta}$ hair cells, we excised utricles and cochleae from $T m c 1^{\Delta / \Delta} T m c 2^{\Delta / \Delta}$ mice at P0-P2 and exposed them to adenoviral $T m c 1^{e x 1}, T m c 1^{e x 2}$, or $T m c 2$ expression vectors with RFP as a transfection marker. We observed robust uptake of FM1-43 in utricles and cochleae exposed to vectors expressing $T m c 2$ (Supplemental Figure 6). In $T m c 1^{\Delta / \Delta} T m c 2^{\Delta / \Delta}$ utricles transfected with $T m c 1^{\text {ex1 }}$, fewer cells displayed robust FM1-43 uptake, consistent with the modest $\operatorname{Tmc} 1^{\text {exl }}$ rescue of mechanotransduction (Figure 7) and the reduced transduction currents in $\mathrm{Tmc1}^{+/+} \mathrm{Tmc} 2^{\Delta / \Delta}$ hair cells (Figure 5). As a control, we transfected $T m c 1^{\Delta / \Delta} T m c 2^{\Delta / \Delta}$ utricles with Ad-Tmc1 $1^{e x 2-d n}$. Although there were many transfected cells, none took up FM1-43, indi- cating that Tmc1- and Tmc2-mediated rescue of FM1-43 uptake in $T m c 1^{\Delta / \Delta} T m c 2^{\Delta / \Delta}$ utricles is a specific effect of expression of functional $T m c 1$ and $T m c 2$.

Gentamicin uptake. To further investigate mechanotransduction in Tmc1; Tmc2 mutant hair cells, we evaluated uptake of gentamicin, a vestibulotoxic and cochleotoxic aminoglycoside antibiotic thought to enter hair cells mainly, if not entirely, through mechanotransduction channels (24). Utricular maculae were explanted from P2 mice, incubated overnight in culture medium, and exposed to $1 \mathrm{mM}$ gentamicin for 48 hours. Wild-type hair bundles were almost completely eliminated (Figure 9, A and B), as previously reported (32). In contrast, hair bundles were intact in gentamicin-treated $T m c 1^{\Delta / \Delta} T m c 2^{\Delta / \Delta}$ hair cells as well as control explants not exposed to gentamicin (Figure 9, A and B). The number of $T m c 1^{+/+} T m c 2^{\Delta / \Delta}$ hair bundles was significantly reduced $(P<0.05,1$-way ANOVA) in comparison to non-exposed controls, whereas $T m c 1^{\Delta / \Delta} T m c 2^{+/+}$ hair bundles were eliminated almost completely (Figure 9, A and B). To confirm uptake of gentamicin in wild-type hair cells and lack of uptake in $T m c 1^{\Delta / \Delta} T m c 2^{\Delta / \Delta}$ hair cells, we stained gentamicinexposed utricles with anti-gentamicin antibodies. After a 6-hour incubation with $1 \mathrm{mM}$ gentamicin, robust gentamicin immunoreactivity was observed in wild-type hair cells, while no gentamicin immunoreactivity was detected in $T m c 1^{\Delta / \Delta} T m c 2^{\Delta / \Delta}$ hair cells (Figure 9C). The lack of gentamicin uptake in Tmc1;Tmc2 mutant utricle hair cells provides another line of evidence supporting the hypothesis that $T m c 1$ or $T m c 2$ is required for vestibular hair cell mechanotransduction.

To examine the uptake of gentamicin in Tmc1;Tmc2 mutant cochlear hair cells, whole cochleae were explanted from P2 mice, incubated overnight in culture medium, exposed to $1 \mathrm{mM}$ gentamicin for 48 hours, and then fixed immediately for visualization. In wild-type and $T m c 1^{\Delta / \Delta} T m c 2^{+/+}$cochleae, both inner and outer hair bundles were eliminated completely, whereas $T m c 1^{\Delta / \Delta}$ $T m c 2^{\Delta / \Delta}$ hair bundles remained (Supplemental Figure 7). Hair cells with functional $T m c 1$ but not $T m c 2\left(T m c 1^{+/+} T m c 2^{\Delta / \Delta}\right)$ showed an intermediate phenotype; some hair bundles were eliminated, but only toward the basal end of the cochlea. To assess whether susceptibility to gentamicin was different at a later developmental stage, we repeated the same experiment on cultures explant- 
A

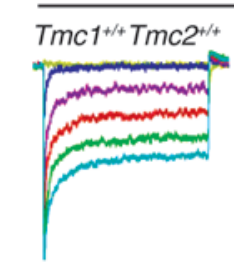

$T m c 1^{\Delta+} T m c 2^{\Delta / \Delta}$

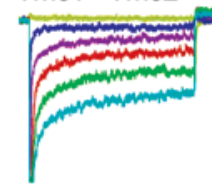

C

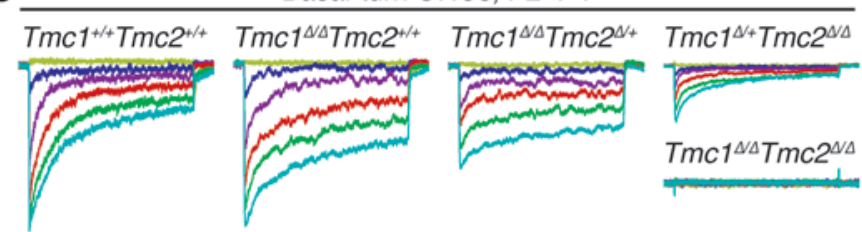

Apical-turn OHCs, P5-P7
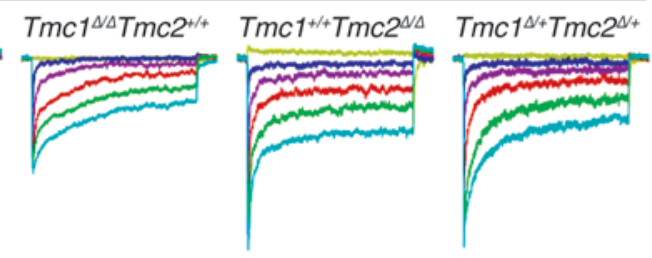

$T m c 1^{\Delta \Delta} T m c 2^{\Delta \Delta}$

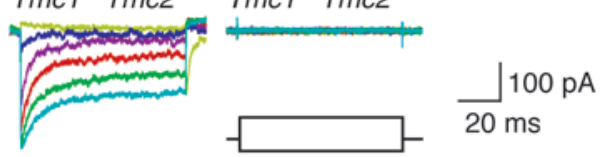

Basal-turn OHCs, P2-P4
B

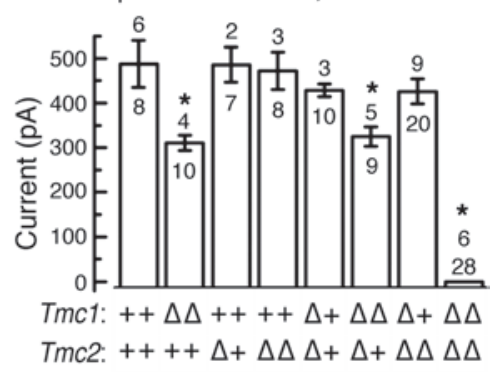

D Basal-turn OHCs, P2-P4

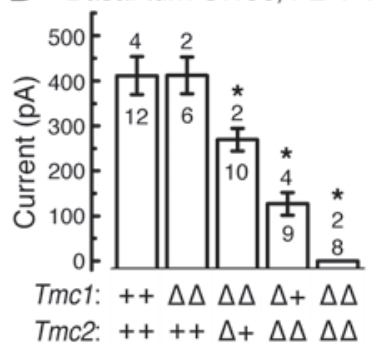

\section{Figure 6}

$T m 1^{\triangle}{ }^{\Delta}$ and $T m c 2^{\Delta}$ cochlear hair cell mechanotransduction currents. (A) Representative mechanotransduction current families recorded from OHCs harvested at P5-P7 from the apical cochlea of mice of the indicated Tmc1;Tmc2 genotypes. Hair bundles were deflected for 90-ms steps that ranged from $200 \mathrm{~nm}$ to $750 \mathrm{~nm}$ in 50-nm increments while cells were held at a physiologically relevant holding potential (-64 $\mathrm{mV}$ ). Some traces have been removed for clarity. The envelope of the stimulus protocol is shown at the bottom right. Tmc1 ${ }^{\Delta / \Delta} \operatorname{Tmc} 2^{\Delta / \Delta} \mathrm{OHCs}$ lack mechanotransduction currents entirely. Scale bars apply to all current families. (B) Summary bar graph of mean maximal mechanotransduction current amplitudes $( \pm$ SEM) from 100 apical OHCs. For each bar, the upper number indicates the number of mice examined, and the lower number indicates the total number of hair cells examined. $T m c 1^{\Delta / \Delta} T m c 2^{+/+}$and $T m c 1^{\Delta / \Delta} T m c 2^{\Delta /+}$ hair cells had intermediate current amplitudes. ${ }^{*} P<0.001$, 1-way ANOVA, relative to wild-type values. (C) Representative transduction currents recorded from OHCs excised at P2-P4 from the basal end of the cochlea from mice of the indicated genotypes. The stimulus protocol and scale bars in A apply. (D) Summary bar graph of maximal mechanotransduction current amplitudes $( \pm$ SEM) from 45 basal OHCs. Upper numbers indicate the numbers of mice examined, and lower numbers indicate the total numbers of cells examined. ${ }^{*} P<0.001,1$-way ANOVA, relative to wild-type values. See also Supplemental Figure 5.

ed at P3 and incubated for 4 days. After a 48-hour incubation in $1 \mathrm{mM}$ gentamicin, similar results were observed. Wild-type hair bundles were eliminated completely, some middle-turn and most basal-turn $T m c 1^{\Delta /+} T m c 2^{\Delta / \Delta}$ hair bundles were eliminated, while $T m c 1^{\Delta / \Delta} T m c 2^{\Delta / \Delta}$ hair bundles remained intact (Supplemental Figure 8). To confirm uptake of gentamicin in wild-type hair cells and no uptake in $T m c 1^{\Delta / \Delta} T m c 2^{\Delta / \Delta}$ hair cells, we stained cochleae with anti-gentamicin antibodies. After a 6-hour incubation with $1 \mathrm{mM}$ gentamicin, robust gentamicin immunoreactivity was observed in wild-type hair cells, while no gentamicin immunoreactivity was detected in $T m c 1^{\Delta / \Delta} T m c 2^{\Delta / \Delta}$ hair cells (Figure 9D). The lack of gentamicin uptake in $T m c 1^{\Delta / \Delta} T m c 2^{\Delta / \Delta}$ hair cells provides another line of functional evidence supporting the hypothesis that Tmc1 or Tmc2 is required for cochlear hair cell mechanotransduction. Moreover, the intermediate loss of hair cells in the basal region of cochleae lacking Tmc2 may reflect the base-to-apex developmental gradient of $T m c 1$ expression at this early postnatal stage (Figure 1B) and the developmental switch from $T m c 2$ to $T m c 1$ expression.

Hair cell localization of TMC proteins. We generated antibodies to TMC1 or TMC2 by in situ immunization of rabbits and in vitro screening of a recombinant human monoclonal antibody library with synthetic peptides and larger recombinant protein fragments corresponding to TMC1 and TMC2. We validated the specificity of antibodies on recombinant TMC1 or TMC2 expressed in heterologous cells, but did not detect specific labeling of hair cells. Our antibodies did not label the lateral membrane of hair cells (data not shown), where potential TMC2 immunoreactivity in the chicken basilar papilla has been observed (33).

As an alternative to immunolocalization of endogenous TMC proteins, we used biolistic gene transfection to express TMC1 ${ }^{\mathrm{Ex} 1}$, $\mathrm{TMC}^{\mathrm{Ex} 2}$, or TMC2 in organotypic cultures of hair cells. TMC proteins were fused at their carboxy termini to AcGFP for visualization. We used either CMV or MYO7A promoters to produce higher or lower levels of expression, respectively. AcGFP did not interfere with the ability of these TMC expression constructs to rescue FM1-43 uptake (data not shown). TMC1 ${ }^{\mathrm{Ex} 1}:$ :AcGFP and TMC1 ${ }^{\mathrm{Ex} 2}:$ :AcGFP expressed from the MYO7A promoter were not detected in stereocilia, but were detected in stereocilia in a few rare experiments using the CMV promoter (Supplemental Figure 9). In contrast, TMC2:: AcGFP was consistently detected near the tips of stereocilia in both vestibular and cochlear hair cells when driven by the MYOTA promoter (Figure 10), but not the CMV promoter (data not shown), consistent with the results of the rescue experiments. 
A

\begin{tabular}{|c|c|c|c|}
\hline Ad-Tmctext & Ad-Tmc1 ${ }^{\times 2}$ & Ad-Tmc2 & $\mathrm{Ad}-T m c 1^{10 \times 2-d n}$ \\
\hline minsins & Tensem & $\mathrm{min}_{\mathrm{m}}$ & $150 \mathrm{pA}$ \\
\hline
\end{tabular}
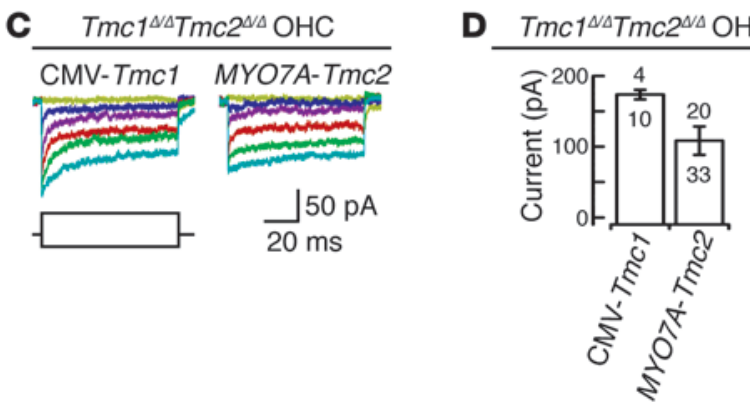

\section{Figure 7}

Rescue of mechanotransduction with Tmc1 or Tmc2. (A) Organotypic cultures of utricles harvested at P0-P3 from $T m c 1^{\Delta \Delta} T m c 2^{\Delta \Delta}$ mice were exposed to adenoviral vectors expressing $T m c 1^{e x 1}(n=4$ mice), $\operatorname{Tmc} 1^{\mathrm{ex} 2}(n=3$ mice), Tmc2 ( $n=5$ mice), or Tmc1ex2-dn $(n=3$ mice) and maintained in culture for 2-4 days. Scale bars apply to all current families. The envelope of the stimulus protocol is shown at the bottom left. (B) For each bar, the upper number indicates the number of cells with measurable mechanotransduction currents, while the lower number indicates total number of tested cells. Mean maximal currents are plotted from cells with measurable current $( \pm$ SEM). (C) Organotypic cultures of cochleae were harvested from $T m c 1^{\Delta / \Delta} T m c 2^{\Delta \Delta}$ mice at P0. Representative mechanotransduction current families for cochlear OHCs transfected with CMV promoter-driven Ad-Tmc1ex1 $(n=4$ mice) or MYO7A promoter-driven Ad-Tmc2 ( $n=11$ mice). Ad-Tmc1 ${ }^{\text {ex1 }}$ or Ad-Tmc2 rescue mechanotransduction in $T m c 1^{\Delta \Delta} T m c 2^{\Delta \Delta}$ OHCs. Scale bars apply to both current families. The envelope of the stimulus protocol is shown at the bottom left. (D) Summary bar graph shows mean maximal mechanotransduction current amplitudes $( \pm$ SEM) from 43 RFP-positive (transfected) OHCs. For each bar, the upper number indicates the number of cells with measurable mechanotransduction currents, while the lower number indicates total number of cells examined.

\section{Discussion}

Our results indicate that TMC1 and TMC2 are necessary for mechanotransduction in early postnatal mouse inner ear hair cells. We suspect that the previously reported mechanotransduction currents in P8 cochlear hair cells from Tmc1 $1^{\text {dn }}$ and $T m c 1^{\text {Bth }}$ mice (9) may have been the result of residual TMC2. Our observation of a developmental switch in the cochlea from Tmc2 to Tmc1 mRNA expression, nearly complete by P8, suggests that TMC2 is not available to compensate for loss of TMC1 in the mature auditory system. This failure to compensate appears to occur at the level of transcription or mRNA stability, since Tmc2 mRNA levels do not increase in $T m c 1^{\Delta / \Delta}$ cochleae (K. Kurima, unpublished observations). Steel and Bock (8) reported that young $d n$ mice (P12-P20), presumably homozygous for the $T m c 1^{d n}$ allele, had no cochlear potentials, a collective measure of hair cell receptor potentials, despite the presence of hair cells. We thus conclude that recessive alleles of TMC1/Tmc1 cause profound deafness via failure to maintain mechanotransduction in mature cochlear hair cells. The loss of transduction in TMC1-deficient mouse cochlear hair cells may ultimately lead to hair cell degeneration, which begins as early as P14, beyond which TMC2 is no longer available to compensate. We propose that the failure of $T m c 1^{d n}$ and $T m c 1^{B t h}$ cochlear hair cells to develop mature voltage-dependent currents at P14 (9) may be an indirect consequence of the failure to maintain mechanotransduction.

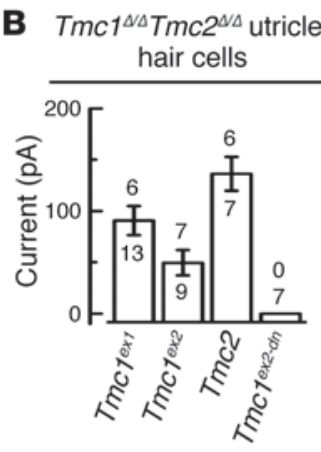

We observed that vestibular hair cells and early postnatal cochlear hair cells lacking TMC1 and TMC2 appear structurally normal in scanning electron micrographs (Figure 4) but have no detectable mechanotransduction (Figures 5 and 6), no FM1-43 uptake (Figure 8), and no gentamicin uptake (Figure 9) at time points when stereocilia and mechanotransduction are well developed in wild-type hair cells $(27,28)$. This structure-function phenotype has not been reported for any other mouse mutants (34) and provides strong genetic evidence for the direct contribution of these proteins to mechanotransduction in inner ear hair cells. A prominent role for TMC2 and TMC1 in inner ear hair cell transduction is further supported by the following experimental observations: First, the rise in Tmc2 mRNA expression in both vestibular and cochlear hair cells coincides with the developmental acquisition of mechanotransduction $(27,28)$, followed $2-3$ days later by a rise in Tmc1 mRNA expression (Figure 1). Second, exogenous expression of either TMC1 or TMC2 can rescue mechanotransduction in either vestibular or cochlear hair cells of $T m c 1^{\Delta / \Delta} T m c 2^{\Delta / \Delta}$ mice (Figure 7). Third, exogenously expressed TMC1 and TMC2 proteins can be localized at or near the tips of stereocilia (Figure 10 and Supplemental Figure 9), as predicted for proteins that directly contribute to mechanotransduction.

Although we did not consistently observe TMC1 protein localization in stereocilia, abundant unpublished data (not shown) suggest that this reflects the technical challenge of expressing sufficient quantities of tagged TMC proteins for detectable localization in stereocilia, without causing a stress response and cell degeneration due to overexpression and retention in the endoplasmic reticulum. These technical challenges associated with TMC localization are consistent with the expected properties of proteins constituting or directly associated with the hair cell mechanotransduction complex.

One hypothesis to explain our results is that TMC1 and TMC2 are required for trafficking, assembly, structure, or function of the mechanotransduction complex. However, since structurally visible components of the complex, such as tip links, remain intact (Figure 4), the missing component remains obscure. An alternate hypothesis that would explain our observations is that TMC1 and TMC2 may be components of the mechanotransduction channel itself $(1,3-5)$. The topology of TMC1 and TMC2 resembles that of a large superfamily of proteins, including Shaker $\mathrm{K}^{+}$and TRP channels, with 6 membrane-spanning segments and cytoplasmic $\mathrm{N}$ and $\mathrm{C}$ termini $(1,5,35,36)$. Moreover, in silico hydropathy analyses imply the existence of two hydrophobic segments that are not predicted to span the membrane and may thus constitute a pore-loop structure (5). These segments are located within the most highly conserved region, the 
A

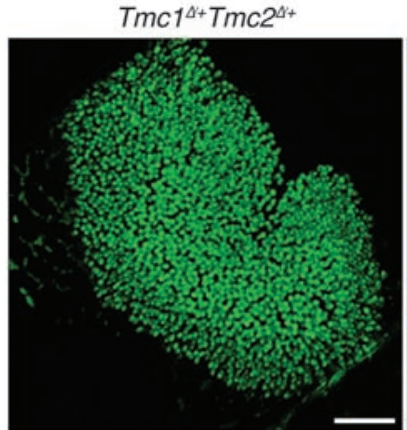

$\mathbf{B}$
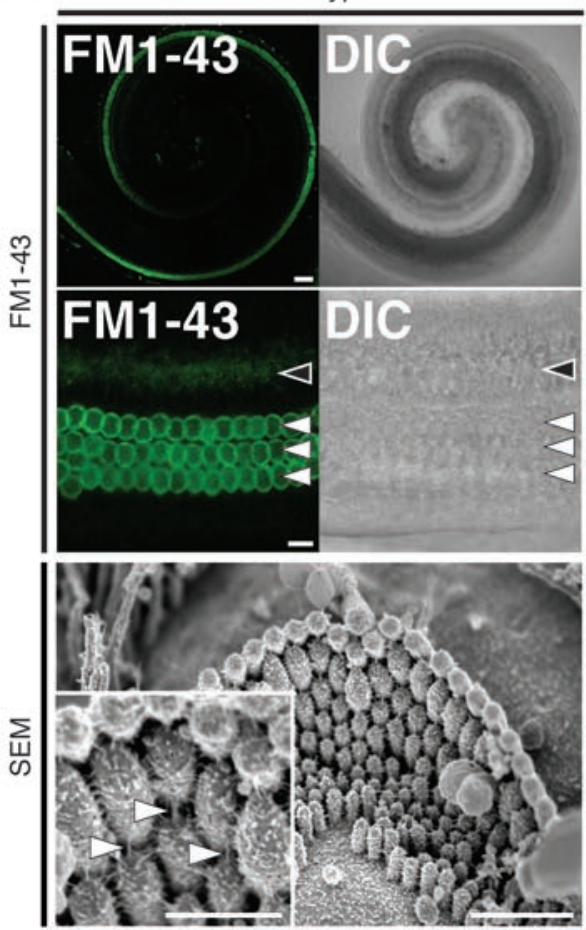

$T m c 1^{\Delta+} T m c 2^{\Delta \Delta}$

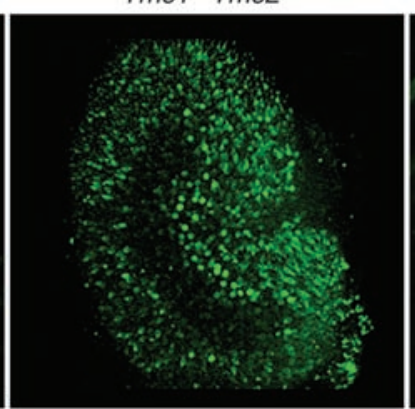

Wild-type + BAPTA
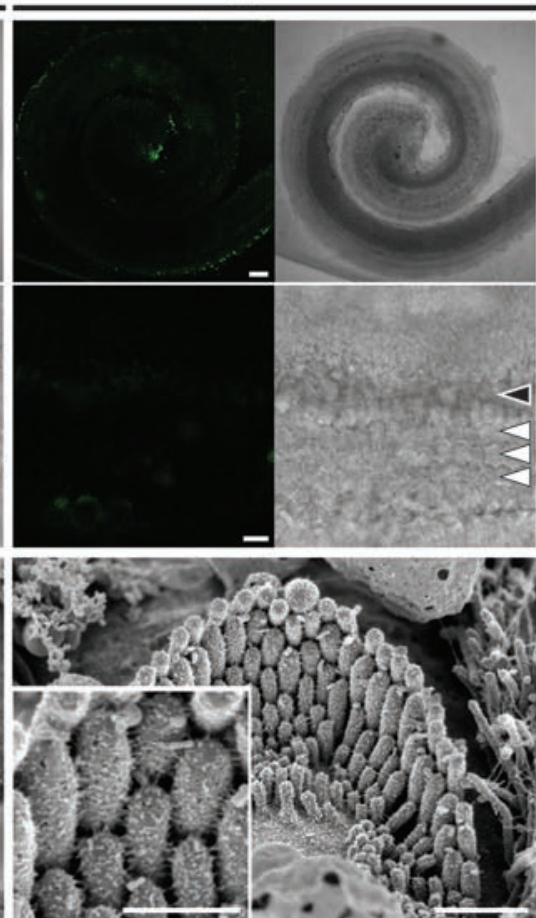

$T m c 1^{\Delta \Delta} T m c 2^{\Delta+}$
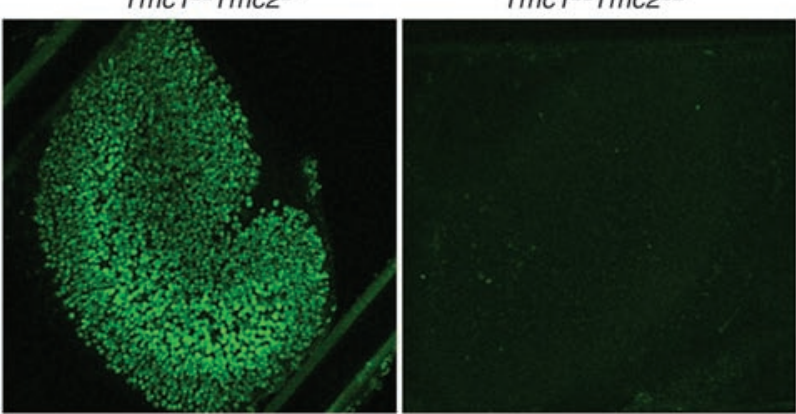

$T m c 1^{\Delta \Delta} T m c 2^{\Delta \Delta}$

\section{Figure 8}

$T m c 1^{\Delta}$ and $T m c 2^{\Delta}$ hair cell uptake of FM1-43. (A) Confocal microscopic images of P3 mouse utricles $\left(T m c 1^{\Delta /+} T m c 2^{\Delta /+}, n=4\right.$ utricles; $T m c 1^{\Delta /+}$ $T m c 2^{\Delta / \Delta}, n=6$; Tmc $1^{\Delta \Delta} T m c 2^{\Delta /+}, n=4 ; T m c 1^{\Delta \Delta} T m c^{\Delta / \Delta}, n=6$ ) exposed to $5 \mu M$ FM1-43. Scale bar: $100 \mu m$. (B) IHCs and OHCs of P3 wild-type mice ( $n=5$ mice) had robust uptake of FM1-43. Wild-type hair cells ( $n=5$ mice) pretreated with 5 mM BAPTA did not take up FM1-43, and tip links are missing (tip links indicated by white arrowheads in insets). No FM1-43 uptake was detected in Tmc $1^{\Delta / \Delta} \operatorname{Tmc} 2^{\Delta / \Delta}$ hair cells $(n=5$ mice) despite intact hair bundles and tip links (compare insets). Black arrowheads, IHCs (middle turn); white arrowheads, OHCs (middle turn); DIC, differential interference contrast. Gamma settings for green channel were adjusted equally throughout entire images and for all images using Adobe Photoshop CS5 software. Scale bars: 100 um, upper panels; $10 \mu \mathrm{m}$, middle panels; 1 um, lower panels; $500 \mathrm{~nm}$, insets in bottom row of panels. See also Supplemental Figure 6.

TMC domain, of TMC proteins $(3,4)$. Part of this domain is deleted in the $\mathrm{Tmcl}^{\text {dn }}$ allele that fails to rescue mechanotransduction (Figure 7, A and B, and Supplemental Figure 6).

Formal proof that TMC1 or TMC2 can constitute the mechanotransduction channel requires data that can directly attribute channel properties to these proteins. Unfortunately, when exogenous TMC1 and TMC2 were expressed in a variety of heterologous systems, they were retained in intracellular compartments and never localized to the plasma membrane, where they could be directly tested for channel activity. We suspect these systems lack hair cell components required for proper trafficking of TMC1 and TMC2 to the plasma membrane of stereocilia.
Altering the intrinsic properties of the hair cell mechanotransduction channel with amino acid substitutions in TMC1 or TMC2 would be another approach to attribute pore-forming channel activity to these proteins. All of the known dominant mutations of $T M C 1 / T m c 1$ are missense amino acid substitutions $(1,2,37,38)$ that must act via a gain-of-function or dominant-negative mechanism (1). The aspartate 572 residue within the highly conserved TMC domain of TMC1 is substituted by two of three reported DFNA36 mutations $(1,37,38)$. The other reported DFNA36 mutation affects the glycine 417 residue that is adjacent to methionine 418 (38), the orthologous residue for methionine 412 that is substituted in $\operatorname{Tmc}^{\text {Bth }}(1,2)$. This clustering of dominant mutations suggests 
A Wild-type

$\mathrm{Tmc1}^{\Delta / \Delta} \mathrm{Tmc2}^{+/+}$

$\mathrm{Tmc}{ }^{+/+} \mathrm{Tmc} 2^{\Delta \Delta}$

$\mathrm{Tmc}^{\mathrm{N}+} \mathrm{Tmc}^{\mathrm{N} \Delta}$

$T m c 1^{\Delta \Delta} T m c 2^{\Delta \Delta}$

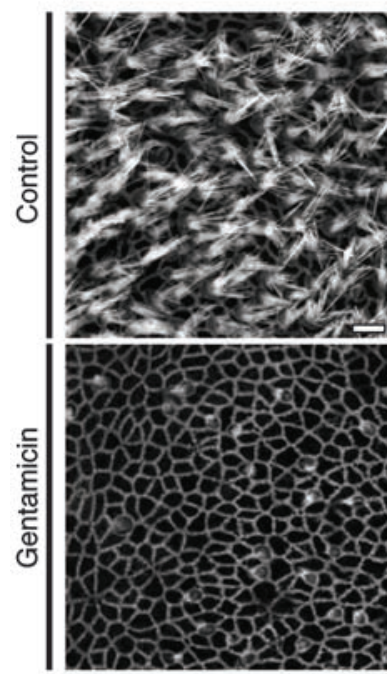

B
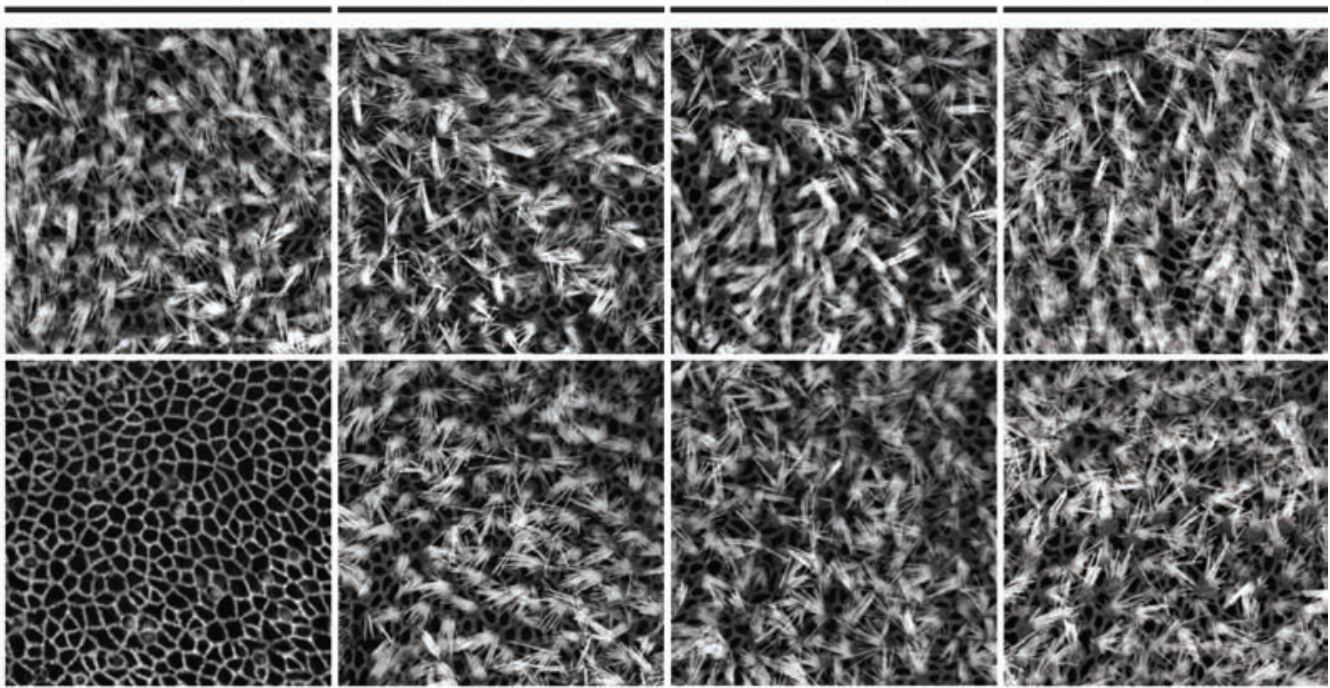

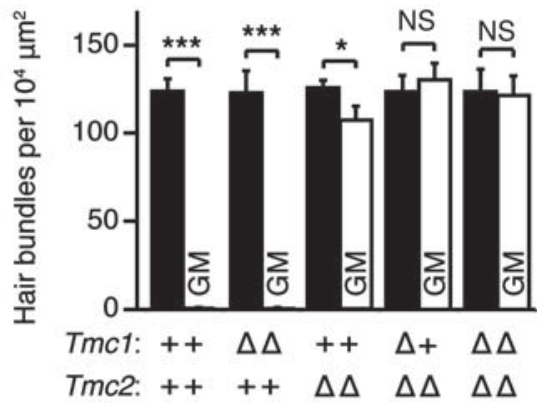

C

Wild-type

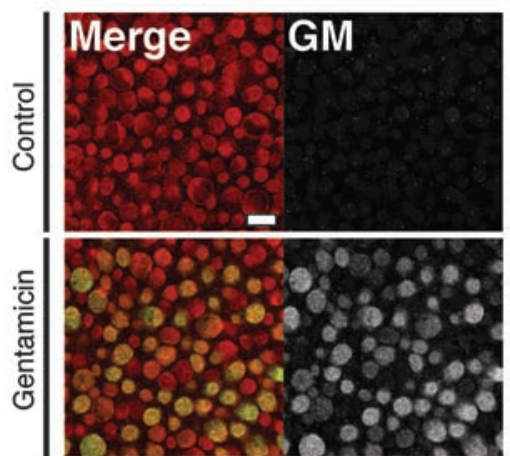

$T m c 1^{\Delta \Delta} T_{m c 2^{\Delta \Delta}}$

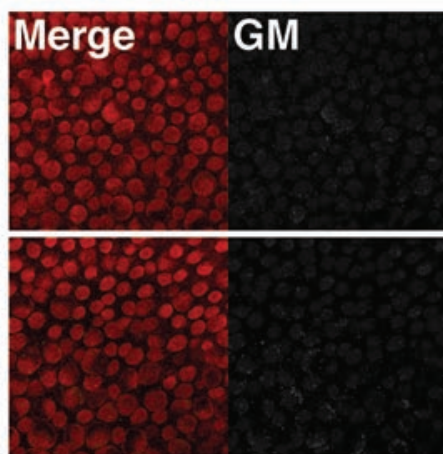

D

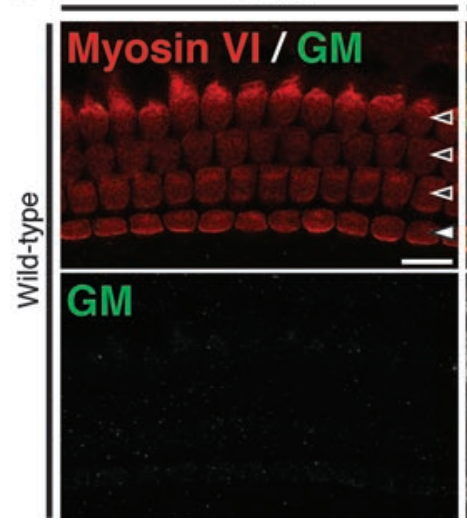

Gentamicin

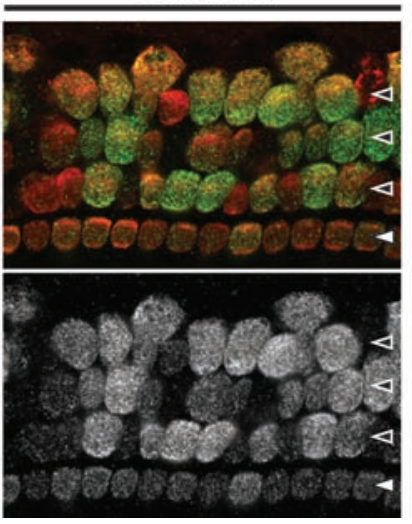

Control

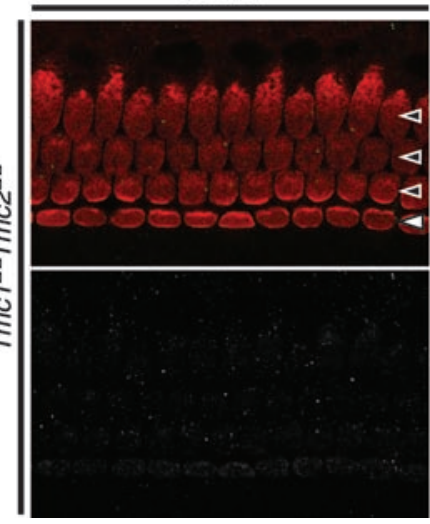

Gentamicin

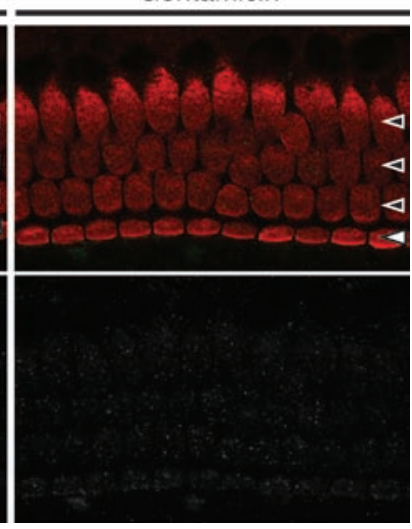

Figure 9

$T m c 1^{\Delta}$ and $T m c 2^{\Delta}$ hair cell uptake of gentamicin. (A) After a 48-hour incubation in culture medium with 1 mM gentamicin, wild-type utricular hair bundles were almost completely eliminated, while $T m c 1^{\Delta / \Delta} T m c 2^{\Delta / \Delta}$ utricular hair cells retained an intact hair bundle. Scale bar: $10 \mu m$. (B) The mean density of intact hair bundles $( \pm S D)$ within the central area of more than 6 utricular maculae of each genotype is shown for each genotype. $T m c 1^{\Delta /+} T m c 2^{\Delta / \Delta}$ and $T m c 1^{\Delta / \Delta} T m c 2^{\Delta / \Delta}$ utricular maculae showed no significant difference between cultures grown with gentamicin in comparison with control cultures grown without gentamicin. $T m c 1^{+/+} T m c 2^{\Delta / \Delta}$ utricular hair cells showed intermediate sensitivity to gentamicin. One-way ANOVA results for differences from normal control values are indicated: ${ }^{*} P<0.05$; ${ }^{\star * \star} P<0.001$; NS, $P>0.05$. (C) After a 6-hour incubation in culture medium with $1 \mathrm{mM}$ gentamicin, robust immunoreactivity for gentamicin was detected in wild-type utricular hair cells, whereas no immunoreactivity for gentamicin was detected in $T m c 1^{\Delta / \Delta} T m c 2^{\Delta / \Delta}$ hair cells. Red, anti-myosin VI antibodies; green, anti-gentamicin antibodies. Scale bar: $10 \mu \mathrm{m}$. (D) After a 6-hour incubation in $1 \mathrm{mM}$ gentamicin, robust immunoreactivity for gentamicin was detected in wild-type IHCs and OHCs, whereas no immunoreactivity for gentamicin was detected in $T m c 1^{\Delta / \Delta} T m c 2^{\Delta / \Delta}$ hair cells. Red, anti-myosin VI antibodies; green, anti-gentamicin antibodies. Filled arrowheads, IHCs; open arrowheads, OHCs. Scale bar: $10 \mu \mathrm{m}$. See also Supplemental Figures 7 and 8. 

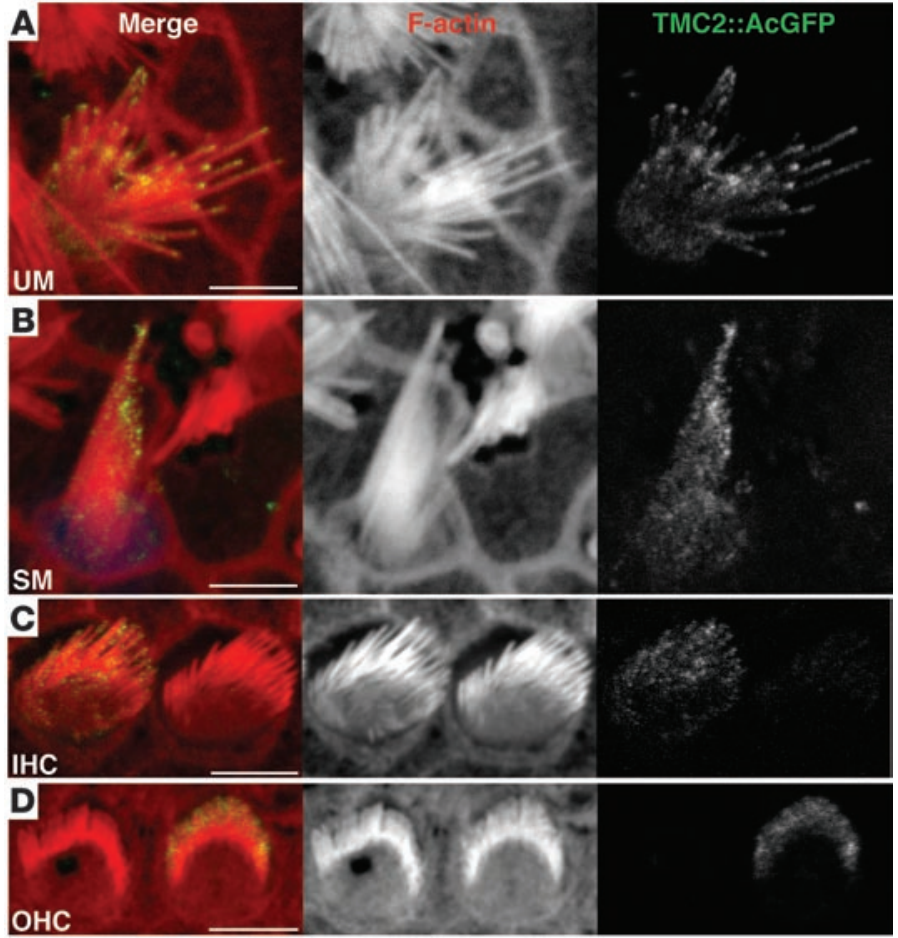

\section{Figure 10}

Localization of TMC2::AcGFP. (A) Rat utricular hair cell transfected with MYO7A-Tmc2::AcGFP at P3 plus 1 day in vitro (P3+1DIV) and incubated for 48 hours. TMC2::AcGFP accumulates toward the tips of stereocilia. Scale bar: $5 \mu \mathrm{m}$. Gamma settings for red and green channels were adjusted equally throughout entire images using Adobe Photoshop CS5. (B) Rat saccular hair cell transfected with MYO7A-Tmc2::AcGFP at P3+1DIV and incubated for 48 hours. TMC2::AcGFP accumulates toward the tips of stereocilia. Scale bar: $5 \mu \mathrm{m}$. (C) $T m c 1^{\Delta / \Delta} T m c 2^{\Delta / \Delta}$ inner hair cells transfected with MYO7A-Tmc2::AcGFP at P0+1DIV and incubated for 72 hours. TMC2::AcGFP expression was detected on stereocilia. Gamma settings for red and green channels were adjusted equally throughout the entire image using Adobe Photoshop CS5. Scale bar: $5 \mu \mathrm{m}$. (D) Rat OHCs transfected with MYO7A-Tmc2::AcGFP at P0+1DIV and incubated for 72 hours. TMC2::AcGFP accumulates toward the tips of stereocilia. Scale bar: $5 \mu \mathrm{m}$. Gamma settings for red and green channels were adjusted equally throughout the entire image using Adobe Photoshop CS5. Cumulative totals of approximately 80 of 100 TMC2::AcGFP-positive hair cells each from cochleae, saccules, and utricles displayed green fluorescence at stereocilia tips. See also Supplemental Figure 9. there is a critical function(s) associated with the wild-type or mutant amino acids at these positions. These mutations are thus excellent candidates to explore potential effects on the intrinsic properties of mechanotransduction. The data could provide insight into the pathogenesis of DFNA36 hearing loss and further evidence supporting the role of TMC proteins in hair cell mechanotransduction.

\section{Methods}

qPCR analysis. Twenty mouse utricular maculae were excised from 10 wildtype mice at each stage between E15 and P21. Ten cochleae were excised from 5 wild-type mice at each age between P0 and P22. RNA was extracted and quality was confirmed using an Agilent Bioanalyzer (Agilent Technologies), and it was reverse transcribed into cDNA for qPCR analysis with efficient primer sets specific to either $T m c 1$ or $T m c 2$ with SYBR GreenER qPCR reagent (Invitrogen) as previously described (39). To amplify a fragment of $T m c 1$ common to both $T m c 1^{e x 1}$ and $T m c 1^{e x 2}$, we used primers $5^{\prime}$-CATCTGCAGCCAACTTTGGTGTGT- ${ }^{\prime}$ ' and $5^{\prime}$-AGAGGTAGCCGGAAATTCAGCCAT-3'. To amplify a fragment of Tmc2, we used primers 5'-AGATCTTTGCGTTCCTTGCCAACC- $3^{\prime}$ and 5'-GATCTTCTTTCGCAGCTGGGCATT- 3 '. Expression levels were normalized to those of $A c t b$ (encoding $\beta$-actin) amplified with 5'-TGAGCGCAAGTACTCTGTGTGGAT-3' and 5'-ACTCATCGTACTCCTGCTTGCTGA-3'. All primers were designed to span introns and validated using melt curve analysis and negative controls.

In situ bybridization analysis. Cryopreserved half-heads between P0 and P5 were sectioned at $12-\mu \mathrm{m}$ thickness and processed for in situ hybridization as described previously (40). Tmc2 transcripts were detected using both $5^{\prime}$ and $3^{\prime}$ RNA probes generated from $T m c 2$ cDNA. The $5^{\prime}$ RNA probe corresponds to exons 2-6, and the $3^{\prime}$ RNA probe corresponds to exons 17-20 of Tmc2.

$T m c 1$ and $T m c 2$ mutant mice. $T m c 1^{\Delta}$ and $T m c 2^{\Delta}$ targeting constructs were designed to replace exons encoding the highly conserved first transmembrane domains (5) (Figure 2, A and B). We generated homology arms from lambda bacteriophage clones encoding Tmc1 exons 8 and 9 or $\operatorname{Tmc} 2$ exon 7 isolated from a 129X1/SvJ mouse genomic library (Agilent Technologies).
Each targeting vector comprised a PPNT plasmid (41) containing Tmc1 or $T m c 2$ homology arms, a gene trap cassette (42), an internal ribosomal entry site (IRES) followed by a lacZ reporter gene, and a neomycin resistance gene (PGK-Neo) flanked by LoxP sites. R1 mouse ES cells (43) were electroporated with NotI-linearized targeting vector and grown in the presence of G418 and ganciclovir, using standard protocols, at the University of Michigan Transgenic Animal Model Core. ES cell clones with proper homologous recombination events were identified by Southern blot analysis, expanded, and injected into C57BL/6J blastocysts. Male chimeras were bred with C57BL/6J females to generate germline $T m c 1^{\Delta}$ or $T m c 2^{\Delta}$ heterozygotes. Germline transmission and Cre recombinase-mediated removal of the PGK-Neo cassette were confirmed by Southern blot (Figure 2, C and D) and PCR analyses (data not shown). Primer information for genotyping is shown in Supplemental Table 1. We ruled out clonal effects by comparison of founder lines derived from two different ES cell clones for both $T m c 1^{\Delta}$ and $T m c 2^{\Delta}$. $T m c 1^{\Delta}$ and $T m c 2^{\Delta}$ lines were backcrossed to C57BL/6J for at least 10 generations to generate congenic lines for all experiments.

lac $Z$ reporter analysis. Otic capsules were dissected from C57BL/6J wild-type, $T m c 1^{\Delta /+}, T m c 2^{\Delta /+}, T m c 1^{\Delta /+} T m c 2^{\Delta / \Delta}$, or $T m c 1^{\Delta / \Delta} T m c 2^{\Delta / \Delta}$ mice ranging from P1 to $\mathrm{P} 28$, and $\beta$-galactosidase activity was detected using X-gal staining (44). Results were confirmed with at least 3 mice of each genotype and age.

Vestibulo-ocular reflex testing. In the absence of CNS or oculomotor abnormalities, the first $100 \mathrm{~ms}$ of the VOR response to high-acceleration wholebody rotations in darkness about an axis through the stereotactic center of the skull and perpendicular to the planes of the horizontal semicircular canals is a sensitive and specific measure of lateral semicircular canal function. Vestibulo-ocular reflex responses to transient yaw rotations were measured (45) in at least 3 adult mice of each genotype at 2-3 months of age (C57BL/6J wild-type, $n=3$; $T m c 1^{\Delta / \Delta} T m c 2^{\Delta /+}, n=3 ; T_{m} 1^{+/+} T m c 2^{\Delta / \Delta}, n=3$; $\left.T m c 1^{\Delta /+} T m c 2^{\Delta / \Delta}, n=3 ; T m c 1^{\Delta / \Delta} T m c 2^{\Delta / \Delta}, n=5\right)$. An array of three fluorescent non-collinear markers was affixed to the anesthetized cornea using cyanoacrylate. Movement of the array was measured using 180 -frame/s, 3-dimensional video-oculography while the mouse's body was rotated about 
an Earth-vertical axis passing through the animal's head with its vision occluded. The stimulus was a constant acceleration of $30,00^{\circ} / \mathrm{s}^{2}$ for $100 \mathrm{~ms}$ and a subsequent cruise at $300 \%$ for $400 \mathrm{~ms}$. Responses were quantified as acceleration gain, the ratio of slow-phase eye velocity to head velocity averaged over the constant acceleration phase of the stimulation. Nystagmus quick phases with high peak velocity $\left(>300^{\circ} / \mathrm{s}\right)$ and peak acceleration $\left(>2,000^{\circ} / \mathrm{s}^{2}\right)$ indicate normal function of the extraocular motor system.

ABR testing. ABR thresholds were measured at 5 or 12 weeks of age in at least 4 mice of each genotype $\left(T m c 1^{+/+} T m c 2^{+/+}, n=5 ; T m c 1^{\Delta /+} T m c 2^{+/+}\right.$, $n=5 ; T m c 1^{\Delta / \Delta} T m c 2^{+/+}, n=5 ; T m c 1^{+/+} T m c 2^{\Delta /+}, n=6 ; T m c 1^{+/+} T m c 2^{\Delta / \Delta}, n=6$; $T m c 1^{\Delta /+} T m c 2^{\Delta /+}, n=9 ; T m c 1^{\Delta / \Delta} T m c 2^{\Delta /+}, n=7 ; T m c 1^{\Delta /+} T m c 2^{\Delta / \Delta}, n=7$; $\left.T m c 1^{\Delta / \Delta} T m c 2^{\Delta / \Delta}, n=4\right)$. We used alternating polarity click and tone-burst stimuli of 5-ms duration (29). Stimulus intensities were initiated at suprathreshold values and initially decreased by $10-\mathrm{dB}$ steps, which were followed by $5-\mathrm{dB}$ steps to determine the ABR threshold. When no ABR waveform was detectable at the highest stimulus level of $100 \mathrm{~dB}$ sound pressure level (SPL), the threshold was considered to be $105 \mathrm{~dB}$ SPL for subsequent analyses.

SEM. Samples were prepared from C57BL/6J wild-type, $T m c 1^{\Delta / \Delta} T m c 2^{\Delta / \Delta}$, $T m c 1^{\Delta / \Delta} T m c 2^{+/+}$, or $T m c 1^{+/+} T m c 2^{\Delta / \Delta}$ mice ranging in age from $\mathrm{P} 0$ to $\mathrm{P} 28$ using the OTOTO method (46) with some modifications (47). Results were confirmed with at least 3 mice of each genotype and age. Otic capsules were fixed in $2.5 \%$ glutaraldehyde buffered with $0.1 \mathrm{M}$ sodium cacodylate containing $2 \mathrm{mM} \mathrm{CaCl}_{2}$ for $1-1.5$ hours at $4{ }^{\circ} \mathrm{C}$, rinsed in $0.1 \mathrm{M}$ sodium cacodylate buffer containing $2 \mathrm{mM} \mathrm{CaCl}_{2}$, and postfixed with $1 \%$ osmium tetroxide $\left(\mathrm{OsO}_{4}\right)$ with $0.1 \mathrm{M}$ sodium cacodylate containing $2 \mathrm{mM} \mathrm{CaCl}_{2}$ for 1 hour at $4^{\circ} \mathrm{C}$. Cochlear and vestibular sensory epithelial tissues were dissected, and the tectorial membrane and otolithic membranes with otoconia were removed in $70 \%$ ethanol. Tissues were gradually hydrated to distilled water, treated with saturated aqueous thiocarbohydrazide (TCH) for 20 minutes, rinsed with distilled water, and immersed in $1 \% \mathrm{OsO}_{4}$ for 1 hour. After 6 washes with $0.1 \mathrm{M}$ sodium cacodylate buffer, the $\mathrm{TCH}$ and $\mathrm{OsO}_{4}$ treatments were repeated twice. The tissues were then gradually dehydrated to ethanol, critical point dried, and imaged with a Hitachi S-4800 field emission electron microscope at 1 - to $10-\mathrm{kV}$ acceleration voltages.

Hair cell electrophysiology. Utricles and cochleae were excised, mounted on glass coverslips, and viewed on an Axioskop FS upright microscope (Zeiss) equipped with a $63 \times$ water-immersion objective and differential interference contrast optics. Electrophysiological recordings were performed at room temperature in solutions containing (in $\mathrm{mM}$ ): $137 \mathrm{NaCl}$, $5.8 \mathrm{KCl}, 10 \mathrm{HEPES}, 0.7 \mathrm{NaH}_{2} \mathrm{PO}_{4}, 1.3 \mathrm{CaCl}_{2}, 0.9 \mathrm{MgCl}_{2}$, and 5.6 D-glucose, vitamins (1:100) and amino acids (1:50) as in MEM (Invitrogen), pH 7.4 $(311 \mathrm{mOsm} / \mathrm{kg})$. Recording electrodes $(2-4 \mathrm{M} \Omega)$ were pulled from R-6 glass (King Precision Glass) and filled with (in mM): $135 \mathrm{KCl}, 5$ EGTA-KOH, 5 HEPES, $2.5 \mathrm{Na}_{2} \mathrm{ATP}, 2.5 \mathrm{MgCl}_{2}$, and $0.1 \mathrm{CaCl}_{2}$, pH 7.4 (284 mOsm/kg). The whole-cell, tight-seal technique was used to record mechanotransduction currents using an Axopatch 200B (Molecular Devices) for utricles and a Multiclamp 700A amplifier (Molecular Devices) for cochleae. Cells were held at $-64 \mathrm{mV}$, a physiologically relevant holding potential. Currents were filtered at $10 \mathrm{kHz}$ with a low-pass Bessel filter, digitized at at least $20 \mathrm{kHz}$ with a 12-bit acquisition board (Digidata 1322A), and recorded using pClamp 8.2 software (Molecular Devices). As previously described (31), hair bundles were deflected using a stiff glass probe mounted on a PICMA chip piezo actuator (Physik Instruments) driven by a $400-\mathrm{mA}$ ENV400 amplifier (Piezosystem Jena) filtered with an 8-pole Bessel filter at $10 \mathrm{kHz}$ to eliminate residual pipette resonance (31).

FM1-43 uptake. Utricles were excised from P3 C57BL/6J wild-type, $T m c 1^{\Delta /+} T m c 2^{\Delta /+}, T m c 1^{\Delta /+} T m c 2^{\Delta / \Delta}, T m c 1^{\Delta / \Delta} T m c 2^{\Delta /+}$, and $T m c 1^{\Delta / \Delta} T m c 2^{\Delta / \Delta}$ mice. $N$-(3-triethylammoniumpropyl)-4-(4-(dibutylamino)styryl)pyridinium dibromide (FM1-43, Invitrogen) was applied to the mounted tissue at $5 \mu \mathrm{M}$ for 10 seconds as previously described (28). Images were obtained with an LSM 510 confocal microscope (Zeiss). Whole cochleae were excised from P3 C57BL/6J wild-type and $T m c 1^{\Delta / \Delta} T m c 2^{\Delta / \Delta}$ mice in Leibovitz's L-15 (L-15) medium and explanted onto a glass-bottom dish (MatTek) previously coated with collagen type I (Millipore). Explants were incubated in DMEM supplemented with $7 \% \mathrm{FBS}$ at $37^{\circ} \mathrm{C}$ with $5 \%$ $\mathrm{CO}_{2}$. Explants were washed 3 times the next morning with HBSS containing $10 \mathrm{mM}$ HEPES buffer and $1.3 \mathrm{mM} \mathrm{Ca}^{2+}$ (HBHBSS-Ca). After an additional 15 minutes incubation in HBHBSS-Ca, $3 \mu \mathrm{M}$ FM1-43 in HBHBSS-Ca was applied for 10 seconds at room temperature, followed immediately by 3 washes ( 30 seconds each) with HBHBSS-Ca. When testing the effects of $5 \mathrm{mM}$ 1,2-bis(2-aminophenoxy)ethane- $N, N, N^{\prime}, N^{\prime}$ tetraacetic acid (BAPTA), HBHBSS-Ca was replaced with $5 \mathrm{mM}$ BAPTA in HBHBSS in every step. FM1-43 fluorescence from whole cochleae was captured with a $10 \times$ objective lens and magnified images from middle turns were captured with a $40 \times$ objective at 5 and 10 minutes after FM143 application, respectively. All images were obtained with identical settings on an LSM 710 confocal microscope equipped with ZEN 2009 software (Zeiss). Gamma settings for the green channel were adjusted equally throughout entire images and for all images using Adobe Photoshop CS5 software. Immediately after imaging, samples were fixed with $2.5 \%$ glutaraldehyde buffered with $0.1 \mathrm{M}$ sodium cacodylate containing $2 \mathrm{mM} \mathrm{CaCl}_{2}$ and prepared for SEM as described above.

Gentamicin uptake. One cochlea or utricle from each experimental mouse was exposed to gentamicin, and the contralateral cochlea or utricle was used as a nonexposed control. Utricular maculae were excised from P2 mice of each genotype (C57BL/6J wild-type, $n=6$; $T m c 1^{\Delta / \Delta} T m c 2^{+/+}$, $n=7 ; T m c 1^{+/+} T m c 2^{\Delta / \Delta}, n=8 ; T m c 1^{\Delta /+} T m c 2^{\Delta / \Delta}, n=6 ; T m c 1^{\Delta / \Delta} T m c 2^{\Delta / \Delta}$, $n=6)$ in L-15 medium and explanted onto a glass-bottom dish (MatTek) previously coated with type I collagen (Millipore). Explants were bathed in DMEM supplemented with 7\% FBS and incubated overnight at $37^{\circ} \mathrm{C}$ with $5 \% \mathrm{CO}_{2}$. Whole cochleae were excised from $\mathrm{P} 2$ or $\mathrm{P} 3$ mice of each genotype and incubated overnight or 4 days, respectively. Numbers of P2 mice were as follows: $T m c 1^{1^{++}} T m c 2^{+/+}, n=6$; $T m c 1^{\Delta / \Delta} T m c 2^{+/+}$, $n=6 ; T m c 1^{+/+} T m c 2^{\Delta / \Delta}, n=6 ; T m c 1^{\Delta /+} T m c 2^{\Delta / \Delta}, n=4 ; T m c 1^{\Delta / \Delta} T m c 2^{\Delta / \Delta}$, $n=3$. Numbers of P3 mice were: $T m c 1^{+/+} T m c 2^{+/+}, n=3 ; T m c 1^{\Delta /+} T m c 2^{\Delta / \Delta}$, $n=3 ; T m c 1^{\Delta / \Delta} T m c 2^{\Delta / \Delta}, n=4$. The culture medium was replaced with fresh gentamicin-free medium for controls or with medium containing $1 \mathrm{mM}$ gentamicin and incubated for 48 hours at $37^{\circ} \mathrm{C}$ with $5 \% \mathrm{CO}_{2}$. The explants were then fixed with $4 \%$ PFA for 30 minutes at room temperature. F-actin was visualized with rhodamine phalloidin (Invitrogen). We counted intact hair bundles within an arbitrarily selected region of $100 \mu \mathrm{m} \times 100 \mu \mathrm{m}$ within the central area of each utricular macula. To more directly evaluate gentamicin uptake into hair cells, additional tissues were explanted and incubated in culture medium containing $1 \mathrm{mM}$ gentamicin for 6 hours at $37^{\circ} \mathrm{C}$ with $5 \% \mathrm{CO}_{2}$, fixed, and stained with mouse monoclonal anti-gentamicin antibodies (QED Bioscience), followed by incubation with goat anti-mouse Alexa Fluor 488 secondary antibodies (Invitrogen). Hair cells were labeled with rabbit polyclonal anti-myosin VI antibodies (Proteus Biosciences) and donkey anti-rabbit Alexa Fluor 568 antibodies (Invitrogen). Images were captured with an LSM 710 confocal microscope equipped with ZEN 2009 software.

$D N A$ expression vectors. Mouse $T m c 1$ and $T m c 2 \mathrm{cDNAs}$ were generated from RNA extracted from inner ear. $T m c 1^{\text {exl }}$ encodes a transcript lacking exon 2, and its translation initiation codon is predicted to be in exon 1 (Supplemental Figure 1A). Tmc $1^{e x 2}$ encodes a transcript including exon 2, and the translation initiation codon is predicted to be in exon 2 (Supplemental Figure

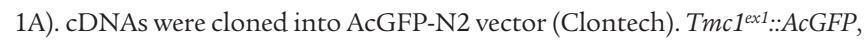
Tmc1 ${ }^{e x 2}:: A c G F P$, and Tmc2::AcGFP cDNA fragments were subcloned into a pAdTrack shuttle plasmid (48) in which the CMV promoter was replaced with a fragment (c. -46 to -3321 ) of the human MYO7A promoter. 


\begin{abstract}
Adenoviral vectors. The coding sequences of $T m c 1^{e x 1}, T m c 1^{e x 2}$, or $T m c 2$ were subcloned into the multiple cloning site of a shuttle vector with a fragment (c. -46 to -3321 ) of the MYO7A promoter. cDNA encoding $T m c 1^{e x 1}$ was subcloned into adenoviral vectors in which expression was driven by a CMV promoter. The vectors also contained a CMV promoter-driven sequence encoding RFP that served as a transfection marker. The resultant plasmid was linearized by digestion with PmeI and subsequently co-transformed into BJ5183 cells with the adenoviral backbone plasmid pAdE $\Delta$ pol (49). Recombinants were selected for kanamycin resistance, and recombination was confirmed by restriction endonuclease analyses. Linearized recombinant plasmids were transfected into C7 cells, an adenovirus packaging cell line (50). For large-scale production, we used serial amplification of crude cell lysate in C7 cells. After 5 rounds of serial passage, the crude lysate was filtered and purified using an AdenoX viral purification kit (BD Biosciences) to yield approximately $2 \mathrm{ml}$ each of Ad-Tmc1 ${ }^{\text {ex1 }}$, Ad-Tmc1 ${ }^{\text {exz }}$, and Ad-Tmc2, at titers that ranged from $10^{7}$ to approximately $10^{9}$ viral particles $/ \mathrm{ml}$, which was distributed into $25-\mu \mathrm{l}$ aliquots and stored at $-80{ }^{\circ} \mathrm{C}$. Viral vectors were added directly to $T m c 1^{\Delta \Delta} T m c 2^{\Delta \Delta}$ organotypic culture medium to final working titers that ranged from $2.5 \times 10^{6}$ to $1 \times 10^{8}$ viral particles $/ \mathrm{ml}$ for $4-24$ hours. The medium was replaced with virus-free medium, and cultures were maintained for an additional 2-4 days.
\end{abstract}

Biolistic transfection. Samples were prepared as described previously (51) for use with the Helios Gene Gun System (Bio-Rad). Gold particles (1.0- $\mu \mathrm{m}$, Bio-Rad) were coated with plasmid DNA at a ratio of $1 \mu \mathrm{g}$ of gold particles to $2 \mu \mathrm{g}$ plasmid DNA encoding Tmc1 ${ }^{\text {ex }}:: A c G F P$, Tmc1 ${ }^{\text {ex2 }}:: A c G F P$, or Tmc2::AcGFP with CMV or MYO7A promoters (see DNA expression vectors above). DNA-coated particles were precipitated onto the inner wall of Tefzel tubing (Bio-Rad), which was cut into individual cartridges containing approximately $1 \mu \mathrm{g}$ of plasmid DNA. Inner-ear sensory epithelium cultures were explanted from P0 or P3 rats. Explants were bathed in DMEM supplemented with 7\% FBS and incubated overnight at $37^{\circ} \mathrm{C}$ with $5 \% \mathrm{CO}_{2}$. Explants were then transfected with a Helios Gene Gun, returned to $37^{\circ} \mathrm{C}$ for $24-72$ hours, and fixed with $3 \%$ paraformaldehyde in PBS (PFA) (51). F-actin was visualized with either rhodamine phalloidin (Invitrogen) or Alexa Fluor 647 phalloidin (Invitrogen). Some samples transfected with CMV-Tmc1 ${ }^{\text {ex2 }}:: A c G F P$ were stained with Alexa Fluor 488-conjugated anti-GFP antibodies (Invitrogen) to facilitate detection of faint GFP expression. Adjacent untransfected hair bundles served as negative controls. Images were captured with an LSM 710 confocal microscope equipped with ZEN 2009 software (Zeiss). Gamma settings for red and green channels were adjusted equally throughout entire images using Adobe Photoshop CS5.

Statistics. Two-tailed Student's $t$ test, with a significance criterion of $P<0.05$, was used to compare acceleration gains of the VOR. One-way ANOVA was used to compare ABR thresholds and maximal mechanotransduction current amplitudes (significance criterion, $P<0.001$ ) and hair bundle densities after gentamicin exposure (significance criterion, $P<0.05$ ).
Study approval. All animal experiments and procedures were performed according to protocols approved by the Animal Care and Use Committee of the National Institute of Neurological Disorders and Stroke/NIDCD, the Animal Care and Use Committee of the Johns Hopkins University School of Medicine, or the Animal Care Committee of the University of Virginia.

\section{Acknowledgments}

We thank Ken Kitamura for support; Charles Askew, Laura Digilio, Geoffrey Horwitz, and Michael Mulheisen for technical assistance; Inna Belyantseva, Jonathan Bird, Felipe Salles, Takashi Sato, and Shin-ichiro Kitajiri for technical advice; Bechara Kachar for helpful discussions; and NIDCD colleagues for critical comments. This work was supported by NIH intramural research funds Z01DC000060-10 (A.J. Griffith) and Z01-DC000021-18 (D.K. Wu); NIH grants R01-DC05439 (J.R. Holt), R01-DC008853 (G.S.G. Géléoc), R01-DC009255 and R01-DC002390 (C.C. Della Santina); and a grant from the Hazel Thorpe Carman \& George Gay Carman Trust for Scientific Research (J.R. Holt). Y. Kawashima was supported in part by Tokyo Medical and Dental University.

Received for publication August 10, 2011, and accepted in revised form October 19, 2011.

Address correspondence to: Andrew J. Griffith, Otolaryngology Branch, National Institute on Deafness and Other Communication Disorders, National Institutes of Health, 5 Research Court, Room 2B-29, Rockville, Maryland 20850-3320, USA. Phone: 301.402.2829; Fax: 301.402.7580; E-mail: griffita@nidcd.nih.gov. Or to: Jeffrey R. Holt, Department of Otolaryngology, Children's Hospital Boston, 300 Longwood Avenue, Center for Life Sciences 12251, Boston, Massachusetts 02115, USA. Phone: 617.919.3574; Fax: 617.919.2771; E-mail: jeffrey.holt@childrens.harvard.edu.

Yoshiyuki Kawashima's present address is: Department of Otolaryngology, Tsuchiura Kyodo General Hospital, Tsuchiura, Japan.

Gwenaëlle S.G. Géléoc's and Jeffrey R. Holt's present address is: Department of Otolaryngology, Children's Hospital Boston, Harvard Medical School, Boston, Massachusetts, USA.

Andrea Lelli's and Yukako Asai's present address is: Unité de Génétique et Physiologie de l'Audition, Institut Pasteur, Paris, France.

Tomoko Makishima's present address is: Department of Otolaryngology, University of Texas Medical Branch, Galveston, Texas, USA.

Valentina Labay's present address is: Otsuka Maryland Medicinal Laboratories Inc., Rockville, Maryland, USA.
1. Kurima K, et al. Dominant and recessive deafness caused by mutations of a novel gene, TMC1, required for cochlear hair-cell function. Nat Genet. 2002;30(3):277-284.

2. Vreugde $S$, et al. Beethoven, a mouse model for dominant, progressive hearing loss DFNA36. Nat Genet. 2002;30(3):257-258.

3. Keresztes G, Mutai H, Heller S. TMC and EVER genes belong to a larger novel family, the TMC gene family encoding transmembrane proteins. BMC Genomics. 2003;4(1):24.

4. Kurima K, Yang Y, Sorber K, Griffith AJ. Characterization of the transmembrane channel-like (TMC) gene family: functional clues from hearing loss and epidermodysplasia verruciformis. Genomics. 2003;82(3):300-308.

5. Labay V, Weichert RM, Makishima T, Griffith AJ Topology of transmembrane channel-like gene 1 protein. Biochemistry. 2010;49(39):8592-8598.

6. de Heer A-MR, et al. Progressive sensorineural hearing loss and normal vestibular function in a Dutch DFNB7/11 family with a novel mutation in TMC1.Audiol Neurotol. 2011;16(2):93-105.

7. Makishima T, Kurima K, Brewer CC, Griffith AJ. Early onset and rapid progression of dominant nonsyndromic DFNA36 hearing loss. Otol Neurotol. 2004;25(5):714-719.

8. Steel KP, Bock GR. The nature of inherited deafness in deafness mice. Nature. 1980;288(5787):159-161. 9. Marcotti W, Erven A, Johnson SL, Steel KP, Kros CJ. $T m c 1$ is necessary for normal functional maturation and survival of inner and outer hair cells in the mouse cochlea. J Physiol. 2006;574(pt 3):677-698.

10. Beurg M, Fettiplace R, Nam JH, Ricci AJ. Localization of inner hair cell mechanotransducer channels using high-speed calcium imaging. Nat Neurosci. 2009;12(5):553-558.

11. Denk W, Holt JR, Shepherd GM, Corey DP. Calcium imaging of single stereocilia in hair cells: localization of transduction channels at both ends of tip links. Neuron. 1995;15(6):1311-1321.

12. Hudspeth AJ. Extracellular current flow and the 
site of transduction by vertebrate hair cells. J Neurosci. 1982;2(1):1-10.

13. Jaramillo F, Hudspeth AJ. Localization of the hair cell's transduction channels at the hair bundle's top by iontophoretic application of a channel blocker. Neuron. 1991;7(3):409-420.

14. Lumpkin EA, Hudspeth AJ. Detection of $\mathrm{Ca}^{2+}$ entry through mechanosensitive channels localizes the site of mechanoelectrical transduction in hair cells. Proc Natl Acad Sci U S A. 1995;92(22):10297-10301.

15. Corey DP, Hudspeth AJ. Response latency of vertebrate hair cells. Biophys J. 1979;26(3):499-506.

16. Crawford AC, Evans MG, Fettiplace R. The actions of calcium on the mechano-electrical transducer current of turtle hair cells. J Physiol. 1991;434:369-398.

17. Geleoc GS, Lennan GW, Richardson GP, Kros CJ. A quantitative comparison of mechanoelectrical transduction in vestibular and auditory hair cells of neonatal mice. Proc Biol Sci. 1997; 264(1381):611-621.

18. Ricci AJ, Crawford AC, Fettiplace R. Tonotopic variation in the conductance of the hair cell mechanotransducer channel. Neuron. 2003;40(5):983-990.

19. Corey DP, Hudspeth AJ. Ionic basis of the receptor potential in a vertebrate hair cell. Nature. 1979;281(5733):675-677.

20. Ricci AJ, Kennedy HJ, Crawford AC, Fettiplace R. The transduction channel filter in auditory hair cells. J Neurosci. 2005;25(34):7831-7839.

21. Farris HE, LeBlanc CL, Goswami J, Ricci AJ. Probing the pore of the auditory hair cell mechanotransducer channel in turtle. J Physiol. 2004; 558(pt 3):769-792.

22. Gale JE, Marcotti W, Kennedy HJ, Kros CJ, Richardson GP. FM1-43 dye behaves as a permeant blocker of the hair-cell mechanotransducer channel. J Newrosci. 2001;21(18):7013-7025.

23. Meyers JR, et al. Lighting up the senses: FM1-43 loading of sensory cells through nonselective ion channels. J Neurosci. 2003;23(10):4054-4065.

24. Marcotti W, van Netten SM, Kros CJ. The aminoglycoside antibiotic dihydrostreptomycin rapidly enters mouse outer hair cells through the mechano-electrical transducer channels. J Physiol. 2005;567(pt 2):505-521.

25. Farris HE, Ricci AJ. Voltage-clamp errors cause anomalous interaction between independent ion channels. Neuroreport. 2005;16(9):943-947.

26. Chalfie M. Neurosensory mechanotransduction. Nat Rev Mol Cell Biol. 2009;10(1):44-52.

27. Geleoc GS, Holt JR. Developmental acquisition of sensory transduction in hair cells of the mouse inner ear. Nat Neurosci. 2003;6(10):1019-1020.

28. Lelli A, Asai Y, Forge A, Holt JR, Géléoc GS. Tonotopic gradient in the developmental acquisition of sensory transduction in outer hair cells of the mouse cochlea. J Neurophysiol. 2009;101(6):2961-2973.

29. Noguchi Y, et al. Multiple quantitative trait loci modify cochlear hair cell degeneration in the Beethoven $\left(T m c 1^{B t h}\right)$ mouse model of progressive hearing loss DFNA36. Genetics. 2006;173(4):2111-2119.

30. Holt JR, Corey DP, Eatock RA. Mechanoelectrical transduction and adaptation in hair cells of the mouse utricle, a low-frequency vestibular organ. J Neurosci. 1997;17(22):8739-8748

31. Stauffer EA, Holt JR. Sensory transduction and adaptation in inner and outer hair cells of the mouse auditory system. J Neurophysiol. 2007;98(6):3360-3369.

32. Taura A, Kojima K, Ito J, Ohmori H. Recovery of hair cell function after damage induced by gentamicin in organ culture of rat vestibular maculae. Brain Res. 2006;1098(1):33-48

33. Mutai H, Mann S, Heller S. Identification of chicken transmembrane channel-like (TMC) genes: expression analysis in the cochlea. Neuroscience. 2005;132(4):1115-1122.

34. Vollrath MA, Kwan KY, Corey DP. The micromachinery of mechanotransduction in hair cells. Annu Rev Neurosci. 2007;30:339-365.

35. MacKinnon R, Cohen SL, Kuo A, Lee A, Chait BT. Structural conservation in prokaryotic and eukaryotic potassium channels. Science. 1998; 280(5360):106-109.

36. Montell C, Birnbaumer L, Flockerzi V. The TRP channels, a remarkably functional family. Cell. 2002;108(5):595-598.

37. Kitajiri S, Makishima T, Friedman TB, Griffith AJ. A novel mutation at the DFNA36 hearing loss locus reveals a critical function and potential genotype-phenotype correlation for amino acid-572 of TMC1. Clin Genet. 2007;71(2):148-152.

38. Yang T, Kahrizi K, Bazazzadeghan N, Meyer N, Najmabadi H, Smith RJ. A novel mutation adjacent to the Bth mouse mutation in the TMC1 gene makes this mouse an excellent model of human deafness at the DFNA36 locus. Clin Genet. 2010;77(4):395-398.

39. Asai Y, Holt JR, Geleoc GS. A quantitative analysis of the spatiotemporal pattern of transient receptor potential gene expression in the developing mouse cochlea. J Assoc Res Otolaryngol. 2010;11(1):27-37.

40. Morsli H, Choo D, Ryan A, Johnson R, Wu DK.
Development of the mouse inner ear and origin of its sensory organs. J Neurosci. 1998;18(9):3327-3335.

41. Tybulewicz VL, Crawford CE, Jackson PK, Bronson RT, Mulligan RC. Neonatal lethality and lymphopenia in mice with a homozygous disruption of the c-abl proto-oncogene. Cell. 1991;65(7):1153-1163.

42. Mountford P, et al. Dicistronic targeting constructs: reporters and modifiers of mammalian gene expression. Proc Natl Acad Sci U S A. 1994; 91(10):4303-4307.

43. Nagy A, Rossant J, Nagy R, Abramow-Newerly W, Roder JC. Derivation of completely cell culturederived mice from early-passage embryonic stem cells. Proc Natl Acad Sci U S A. 1993;90(18):8424-8428.

44. Ben-Yosef T, et al. Claudin 14 knockout mice, a model for autosomal recessive deafness DFNB29, are deaf due to cochlear hair cell degeneration. Hum Mol Genet. 2003;12(16):2049-2061.

45. Migliaccio AA, Meierhofer R, Della Santina CC. Characterization of the $3 \mathrm{D}$ angular vestibuloocular reflex in C57BL6 mice. Exp Brain Res. 2011; 210(3-4):489-501.

46. Hunter-Duvar IM. A technique for preparation of cochlear specimens for assessment with the scanning electron microscope. Acta Otolaryngol Suppl. 1978;351:3-23.

47. Lelli A, Kazmierczak P, Kawashima Y, Muller U, Holt JR. Development and regeneration of sensory transduction in auditory hair cells requires functional interaction between cadherin-23 and protocadherin-15. J Neurosci. 2010;30(34):11259-11269.

48. Luo J, et al. A protocol for rapid generation of recombinant adenoviruses using the AdEasy system. Nat Protoc. 2007;2(5):1236-1247.

49. Hodges BL, Serra D, Hu H, Begy CA, Chamberlain JS, Amalfitano A. Multiply deleted [E1, polymerase-, and PTP-] adenovirus vector persists despite deletion of the preterminal protein. J Gene Med. 2000;2(4):250-259.

50. Amalfitano A, Hauser MA, Hu H, Serra D, Begy $\mathrm{CR}$, Chamberlain JS. Production and characterization of improved adenovirus vectors with the E1, E2b, and E3 genes deleted. J Virol. 1998; 72(2):926-933.

51. Belyantseva IA. Helios Gene Gun-mediated transfection of the inner ear sensory epithelium. Methods Mol Biol. 2009;493:103-123.

52. Anderson DW, et al. The motor and tail regions of myosin XV are critical for normal structure and function of auditory and vestibular hair cells. Hum Mol Genet. 2000;9(12):1729-1738. 